\title{
On the Power of Symmetric Linear Programs
}

\author{
Albert Atserias $^{1} \quad$ Anuj Dawar ${ }^{2} \quad$ Joanna Ochremiak $^{2}$ \\ Universitat Politècnica de Catalunya ${ }^{1}$ \\ University of Cambridge ${ }^{2}$
}

January 24, 2019

\begin{abstract}
We consider families of symmetric linear programs (LPs) that decide a property of graphs (or other relational structures) in the sense that, for each size of graph, there is an LP defining a polyhedral lift that separates the integer points corresponding to graphs with the property from those corresponding to graphs without the property. We show that this is equivalent, with at most polynomial blow-up in size, to families of symmetric Boolean circuits with threshold gates. In particular, when we consider polynomial-size LPs, the model is equivalent to definability in a non-uniform version of fixed-point logic with counting (FPC). Known upper and lower bounds for FPC apply to the non-uniform version. In particular, this implies that the class of graphs with perfect matchings has polynomial-size symmetric LPs while we obtain an exponential lower bound for symmetric LPs for the class of Hamiltonian graphs. We compare and contrast this with previous results (Yannakakis 1991) showing that any symmetric LPs for the matching and TSP polytopes have exponential size. As an application, we establish that for random, uniformly distributed graphs, polynomial-size symmetric LPs are as powerful as general Boolean circuits. We illustrate the effect of this on the well-studied planted-clique problem.
\end{abstract}




\section{Introduction}

The theory of linear programming is a powerful and widely-used tool for studying combinatorial optimization problems. By the same token, the limitations of such methods are an important object of study in complexity theory. A major step in this line of work was the seminal paper of Yannakakis [40] that initiated the study of symmetric linear programs for combinatorial problems.

A polytope in $\mathbb{R}^{n}$ is the convex hull of a finite set of points in $\mathbb{R}^{n}$. Dually, it is the intersection of the finite number of half-spaces that define its facets. Consider a language $S \subseteq\{0,1\}^{*}$ and let $S_{n} \subseteq\{0,1\}^{n}$ be the collection of strings in $S$ of length $n$. We can associate with $S_{n}$ the polytope $P_{n} \subseteq \mathbb{R}^{n}$ that is the convex hull of the points $\mathbf{x} \in \mathbb{R}^{n}$ with 0-1 coordinates that correspond to the strings in $S_{n}$. If this polytope has a succinct representation as a system of linear inequalities, we can use linear programming methods to optimize linear functions over $S_{n}$. In general, a succinct representation might mean that its size grows polynomially with $n$. Thus, the size of the polytope $P_{n}$, say measured by the number of its facets, is an important measure of the complexity of $S$.

In general, even when $P_{n}$ has a large number of facets, it may admit a succinct representation as the projection onto $\mathbb{R}^{n}$ of a polytope $Q \subseteq \mathbb{R}^{n+m}$ of higher dimension. In this situation, we call $Q$ a lift of $P_{n}$ and $P_{n}$ a shadow of $Q$. This is the basis for so-called extended formulations of combinatorial optimization problems. It allows us to optimize over $S_{n}$ using linear programs with auxiliary variables. A classic example is the convex hull of all strings in $\{0,1\}^{n}$ of odd Hamming weight, known as the parity polytope which has exponentially many facets but has an extended formulation using only polynomially many inequalities. An interesting feature of many such examples of small extended formulations is that they are strongly symmetric, i.e., any basic automorphism of the shadow polytope extends to an automorphism of its lift.

Yannakakis [40] established lower bounds on the size of symmetric lifts for the perfect matching polytope and the travelling salesman polytope. The perfect matching polytope on $2 n$ vertices is the convex hull of points in $\{0,1\}^{E}$ where $E=\left(\begin{array}{c}{[2 n]} \\ 2\end{array}\right)$ which represent the edge sets of a perfect matching on $2 n$ vertices. Yannakakis shows that any symmetric lift $Q$ of this polytope necessarily has a number of facets that is exponential in $n$. Here "symmetric" means that any permutation of the $n$ vertices extends to an automorphism of $Q$. This lower bound is then used to show a similar lower bound for symmetric lifts of the Hamilton cycle polytope (also known as the travelling salesman polytope). This is the convex hull of points in $\{0,1\}^{E}$ where $E=\left(\begin{array}{c}{[n]} \\ 2\end{array}\right)$ which are the edge sets of Hamilton cycles of length $n$. The conclusion is that any attempt to solve the travelling salesman problem by representing it as a linear program in a natural way (i.e. respecting the symmetries of the graph) is doomed to be exponential. These results launched a long study of extended formulations of combinatorial problems. Relatively recently, exponential lower bounds have been established even without the assumption of symmetry [35].

There is another way of representing a language $S \subseteq\{0,1\}^{*}$ by a family of polytopes that is also considered by Yannakakis. Say that $S_{n}$ is recognized by a polytope $P_{n}$ if $S_{n} \subseteq P_{n}$ and $\{0,1\}^{n} \backslash S_{n}$ is disjoint from $P_{n}$. In particular, the convex hull of $S_{n}$ recognizes $S_{n}$, but 
it may well be that there are more succinct polytopes that also do. Indeed, Yannakakis shows that for any language $S$ decidable in polynomial time, there is a family of polynomialsize polytopes whose shadows recognize $S_{n}$. Thus, we cannot expect to prove exponential lower bounds on such polytopes without separating P from NP. Note that the assumption of symmetry has been dropped here. What can we say about symmetric lifts of polytopes recognizing $S_{n}$ ? Yannakakis does not consider this question and it does not appear to have been studied in the literature. This is the question that we take up in this paper.

We consider families of symmetric polytopes for recognizing classes of graphs (or other relational structures). This gives an interesting contrast with the results of Yannakakis. Our results show that there is a polynomial-size family of symmetric polytopes whose shadows recognize the class of graphs that contain a perfect matching. On the other hand, there is no family of symmetric polytopes of sub-exponential size whose shadows recognize the class of graphs with a Hamiltonian cycle.

We obtain these specific upper and lower bounds by relating the power of symmetric linear programs to two other natural models of symmetric computation, based respectively on logic and circuits. To be precise, we show that families of symmetric polytope lifts for recognizing a class of structures are equivalent to families of symmetric Boolean circuits with threshold gates, in the sense that there are translations between them with at most a polynomial blowup in size in either direction. This places symmetric linear programs squarely in the context of a fairly robust notion of symmetric computation that has recently emerged. It was shown in [3] that P-uniform families of symmetric circuits with threshold gates are equivalent to fixed-point logic with counting (FPC), a well-studied logic in descriptive complexity theory (see [14]).

Our translation from circuits to linear programs is based on that given by Yannakakis, but we need to preserve symmetry and, for threshold gates, this poses a significant challenge. To construct symmetric linear programs that enforce the values of threshold gates we need a sweeping generalization of the construction of symmetric lifts of the parity polytope. In the other direction, we make a detour through logic. That is, we show how a family of symmetric polytopes can be translated into a family of formulas of first-order logic with counting, with the number of variables and the size being tightly bounded based on the size of the polytopes. The translation is based on a support theorem, which allows us to interpret in the logic, given a linear program $P$ as advice, a version of $P$ for a particular input structure. This then allows us to use the result of [4] to the effect that solvability of linear programs is definable in FPC.

It is interesting to compare our results with the equivalence between FPC and P-uniform symmetric threshold circuits established in [3]. Our results are stated for the non-uniform model and it is not clear that they can be made uniform. In particular, our translation from linear programs to formulas of counting logic, while it preserves size, is not necessarily computable in polynomial time. It involves symmetry checks that are as hard as the graph isomorphism problem. On the other hand, the results in [3] were stated for polynomial-size families of circuits and we are able to extend them to sizes up to weakly exponential. The translation from circuits to formulas given in [3] was based on a support theorem proved there 
which only worked for circuit sizes bounded by $O\left(2^{n^{1 / 3}}\right)$. We use a stronger support theorem (proved in Section 4.2) which enables us to prove the translation from families of symmetric linear programs to formulas of counting logic for sizes up to $O\left(2^{n^{1-\epsilon}}\right)$ for arbitrarily small $\epsilon$.

The upper and lower bounds for symmetric linear programs that we obtain (such as for the perfect matching and the Hamilton cycle problem, respectively) are direct consequences of the equivalence with non-uniform counting logic. For instance, it is known [4 that perfect matching is definable in FPC and it follows that it is recognized by a polynomial-size family of symmetric polytope lifts. Inexpressibility results for FPC are usually established by showing lower bounds on the number of variables required to express a property in counting logic, and they yield lower bounds even in the non-uniform setting. In particular, we tighten known lower bounds on Hamiltonicity to show that it cannot be expressed with a sub-linear number of variables hence with weakly exponential size symmetric polytope lifts. Indeed, if we use the strongest form of our translation from symmetric LPs to logic formulas, then the lower bound we get for Hamiltonicity is even exponential, i.e., of type $2^{\Omega(n)}$ where $n$ is the number of vertices of the graph. Similar exponential lower bounds for other NPcomplete problems (such as graph 3-colourability and Boolean satisfiability) follow from known bounds in counting logic. Indeed, exponential lower bounds for some problems in $\mathrm{P}$ (such as solving systems of linear equations over finite fields) also follow. It should be noted that this establishes exponential lower bounds also on symmetric threshold circuits, a problem left open in [3], where superpolynomial lower bounds were established.

Another consequence can be derived from the connection with FPC. We know that FPC can express all polynomial-time properties of almost all structures under a uniform distribution (see [23]). This can be used to show that FPC can solve the planted clique problem if, and only if, the problem is solvable in polynomial time. The planted clique problem is that of distinguishing a random graph from one in which a clique has been planted. It is a widely studied problem in the context of lower bounds on linear programming methods (see e.g. [1, 19, 7, 26]). It is a consequence of our results that if this problem can be solved in polynomial time, then it is solvable by polynomial sized symmetric linear programs. This is significant because a number of lower bounds have been established for the planted clique problem for a variety of models of linear and semidefinite programming, notably the well-studied Lovász-Schrijver, Sherali-Adams and Lasserre hierarchies. It is noteworthy that all of these hierarchies yield symmetric linear or semidefinite programs. Our results show that these lower bounds cannot be extended to general symmetric linear programs without separating $\mathrm{P}$ from NP.

In Section 2 we establish some preliminary definitions and notation. Section 3 gives the translation of circuits to linear programs. This translation is carried out for a very general notion of symmetry. For the reverse translation, from linear programs to logic given in Section 4, we restrict to the natural symmetries on graphs and relational structures. The main result and its consequences, including upper and lower bounds are presented in Section 5 , 


\section{Preliminaries}

For a natural number $n \in \mathbb{N}$, we write $[n]$ for $\{1, \ldots, n\}$ with the understanding that $[0]=\emptyset$. For any set $X$, by $\operatorname{Sym}_{X}$ we denote the symmetric group on $X$, that is, the group of all permutations of the set $X$, and by $\operatorname{Alt}_{X}$ we denote the alternating group on $X$, that is, the group of all even permutations of the set $X$. In the special case of $X=[n]$ we write $\operatorname{Sym}_{n}$ and $\mathrm{Alt}_{n}$, respectively. Logarithms are base 2 with the convention that $\log (0)=0$.

\subsection{Group actions}

By $H \leq G$ we denote the fact that $H$ is a subgroup of $G$. If $H \leq G$ then, for any $g \in G$, the subset $g H=\{g h: h \in H\}$ of $G$ is called a coset of $H$ in $G$. The number of such cosets is called the index of $H$ in $G$ and is denoted by $[G: H]$.

Recall that for any group $G$, a $G$-set is a set $X$ with an action of the group $G$, where by an action we mean a mapping $: G \times X \rightarrow X$ such that for any $\pi, \sigma \in G$ and any $x \in X$ we have $\pi \cdot(\sigma \cdot x)=\pi \sigma \cdot x$ and id $\cdot x=x$. Equivalently, $X$ is a $G$-set if it comes with a homomorphism from the group $G$ to the symmetric group $\operatorname{Sym}_{X}$ on $X$. A homomorphism from a $G$-set $X$ to a $G$-set $Y$ is a function $f$ from $X$ to $Y$ such that for any $\pi \in G$ and $x \in X$, it holds that $\pi \cdot f(x)=f(\pi \cdot x)$. For a $G$-set $X$, a stabilizer of an element $x \in X$ in $G$ consists of all $\pi \in G$ such that $\pi \cdot x=x$. It is easy to see that a stabilizer is a subgroup of $G$. We sometimes denote it by $G_{x}$.

For any set $X$, by $|X|$ we denote the number of elements in $X$, by $\mathcal{P}(X)$ we denote the power set of $X$ and by $X^{n}$ we denote the set of $n$-tuples of elements of $X$. Moreover, if $n \leq|X|$, by $X^{(n)}$ we denote the set of all $n$-tuples of distinct elements of $X$. In particular, for $n=0$, both $X^{n}$ and $X^{(n)}$ are one-element sets consisting of the empty tuple. If $X$ is a $G$-set, then the action of $G$ on $X$ induces an action of $G$ on each of the sets $\mathcal{P}(X), X^{n}$ and $X^{(n)}$ in the natural way: for any $\pi \in G$, we have $\pi \cdot T=\{\pi \cdot x: x \in T\}$, where $T \subseteq X$, and $\pi \cdot \mathbf{s}=\left(\pi \cdot s_{1}, \ldots, \pi \cdot s_{n}\right)$, where $\mathbf{s}=\left(s_{1}, \ldots, s_{n}\right)$ is a tuple from $X^{n}$ or $X^{(n)}$. We refer to the latter group action as the componentwise action of the group $G$.

An action of a group $G$ on a set of indices $U$ defines an action of $G$ on the set of indexed variables $\left\{x_{u}\right\}_{u \in U}$ in the natural way: $\pi \cdot x_{u}=x_{\pi \cdot u}$, for any $\pi \in G$ and $u \in U$. This extends to vectors of indexed variables, as discussed in the paragraph above. For instance, if the set of indices $[n]^{2}$ comes with the componentwise action of the group $\mathrm{Sym}_{n}$, then for any $\pi \in \operatorname{Sym}_{n}$ and $\mathbf{x}=\left(x_{i j}\right)_{i, j \in[n]}$, we have $\pi \cdot \mathbf{x}=\left(x_{\pi(i) \pi(j)}\right)_{i, j \in[n]}$. From now on, in the case of vectors of variables we use the notation $\mathbf{x}^{\pi}$ instead of $\pi \cdot \mathbf{x}$.

For any $G$-set $U$, we define an action of $G$ on the real vector space $\mathbb{R}^{U}$ in the following way. First, the action of $G$ on the standard basis $\left\{e_{u}\right\}_{u \in U}$, where $e_{u}$ is the vector whose $u$-th coordinate is 1 and all other coordinates are 0 , is given by $\pi \cdot e_{u}=e_{\pi \cdot u}$, for any $\pi \in G$ and $u \in U$. This way each $\pi \in G$ defines a mapping from $\left\{e_{u}\right\}_{u \in U}$ to $\left\{e_{u}\right\}_{u \in U}$. The action of $G$ on the vector space spanned by $\left\{e_{u}\right\}_{u \in U}$ can be seen as the linear extension of those mappings: for any $\pi \in G$ and any real vector $\mathbf{a}=\sum_{u \in U} a_{u} e_{u}$, we have $\pi \cdot \mathbf{a}=$ $\sum_{u \in U} a_{u}\left(\pi \cdot e_{u}\right)=\sum_{u \in U} a_{u} e_{\pi \cdot u}$. For instance, if the set of indices [n] comes with the natural action of the group $\operatorname{Sym}_{n}$, then for any $\pi \in \operatorname{Sym}_{n}$ and for any vector $\mathbf{a}=\left(a_{1}, \ldots, a_{n}\right)$, we 
have $\pi \cdot \mathbf{a}=\sum_{i \in[n]} a_{i} e_{\pi(i)}=\sum_{i \in[n]} a_{\pi^{-1}(i)} e_{i}=\left(a_{\pi^{-1}(i)}, \ldots, a_{\pi^{-1}(n)}\right)$. Here again we use the notation $\mathbf{a}^{\pi}$ instead of $\pi \cdot \mathbf{a}$. This notational convention extends to subsets of real vector spaces: for $P \subseteq \mathbb{R}^{U}$ we write $P^{\pi}$ instead of $\pi \cdot P$.

If a group $G$ acts on a set $U$ and a group $H$ acts on a set $W$, then the product group $G \times H$ acts on the disjoint union $U \dot{\cup} W$ : given $\pi \in G$ and $\sigma \in H$, we have $(\pi, \sigma) \cdot u=\pi \cdot u$, for $u \in U$, and $(\pi, \sigma) \cdot w=\sigma \cdot w$, for $w \in W$. Given such an action of the product group $G \times H$, of particular interest to us is its induced action on $\mathbb{R}^{U} \times \mathbb{R}^{W}$ and on sets of variables indexed by $U \dot{\cup} W$.

\subsection{Logic and structures}

A (many-sorted relational) vocabulary consists of a finite set of sort symbols and a finite set of relation symbols. Each relation symbol $R$ comes with an associated natural number $\operatorname{ar}(R)$ called its arity and with an associated type which is a product of $\operatorname{ar}(R)$-many sort symbols $U_{i_{1}} \times \ldots \times U_{i_{\operatorname{ar}(R)}}$. The vocabulary $L_{G}$ of (directed) graphs is single-sorted and has one relation symbol $E$ of arity two. If $L$ is a vocabulary, then an $L$-structure $\mathbb{A}$ is given by disjoint sets $U_{1}, \ldots, U_{s}$, called domains, one for each sort symbol in $L$, and a relation $R^{\mathbb{A}} \subseteq U_{i_{1}} \times \ldots \times U_{i_{r}}$ for each $R \in L$ of arity $r$ and type $U_{i_{1}} \times \ldots \times U_{i_{r}}$. The relation $R^{\mathbb{A}}$ is called the interpretation of $R$ in $\mathbb{A}$. Whenever this does not lead to confusion we use $U$ to denote the domain associated to the sort symbol $U$. Moreover, when $\mathbb{A}$ is clear from the context, we omit the superscript in $R^{\mathbb{A}}$. All our structures are finite: their domains are finite sets. A directed graph is an $L_{G}$-structure; the graph is undirected if its interpretation of $E$ is symmetric and irreflexive.

In a logic for a many-sorted vocabulary $L$ the variables are typed, that is, each different sort has its own set of individual variables. When an $L$-formula is interpreted on an $L$ structure, the variables range over the domain of their sort. The atomic $L$-formulas are equalities between variables of the same type, and formulas of the form $R\left(x_{1}, \ldots, x_{r}\right)$, where $R$ is a relation symbol of arity $r$ in $L$, and $x_{1}, \ldots, x_{r}$ are variables of appropriate types.

The class of formulas of first-order logic (FO) is the smallest class of formulas that contains all atomic formulas and is closed under negation, conjunction, and existential quantification. We consider an extension of first-order logic with counting quantifiers. For each natural number $q$, we have a quantifier $\exists^{\geq q}$ where $\mathbb{A} \models \exists^{\geq q} x \phi$ if, and only if, there are at least $q$ distinct elements $a \in A$ such that $\mathbb{A} \models \phi[a / x]$. While the extension of first-order logic with counting quantifiers is no more expressive than FO itself, the presence of these quantifiers does affect the number of variables that are necessary to express a query. Let $\mathrm{C}^{k}$ denote the $k$-variable fragment of this logic, i.e. those formulas in which no more than $k$ variables appear, free or bound. FPC is the extension of first-order logic with fixed-points and counting. We do not give a full definition here as it can be found in standard texts such as [33]. We note that formulas of FPC have two sorts of variables, ranging respectively over the elements of the domain of interpretation and over natural numbers (restricted to the size of the domain), and allow for terms of the form $\# x \phi$ which denotes the number of elements that satisfy $\phi(x)$. We write FOC to denote the fragment of FPC without fixed-point operators, but where we do allow arithmietic operations $(+$ and $\times$ ) on the number sort. The size of a formula is 
defined as the number of its subformulas. For each formula $\phi$ of FPC (and, per force, FOC), if the formula uses $k$ variables then, for every $n$, there is a formula $\theta_{n}$ of $\mathrm{C}^{2 k}$ such that $\phi$ is equivalent to $\theta_{n}$ on all structures of size at most $n$. Moreover, $\theta_{n}$ has size that is polynomial in the size of $\phi$, in $k$, and in $n^{k}$. For more on FPC and its relation to the bounded variable fragments $\mathrm{C}^{k}$ we refer to 33 .

Rational numbers $q \in \mathbb{Q}$ are represented by structures of a single-sorted vocabulary $L_{\mathbb{Q}}$ with three monadic relation symbols and one binary relation symbol $\leq$. If $q=(-1)^{b} n / d$, where $n, d \in \mathbb{N}$ and $b \in\{0,1\}$, then the domain of an $L_{\mathbb{Q}^{-s t r u c t u r e ~ t h a t ~ r e p r e s e n t s ~} q \text { is }}$ $\{0, \ldots, N\}$ where $N \in \mathbb{N}$ is large enough to represent both the numerator and denominator with $N$ bits. The binary relation $\leq$ is interpreted by the natural linear order on $\{0, \ldots, N\}$. The first of the monadic relation symbols of $L_{\mathbb{Q}}$ is used to represent the sign $b$ of $q$ by having it empty if, and only if, $b=0$. The other two monadic relation symbols of $L_{\mathbb{Q}}$ are used to represent the bit positions on which the numerator $n$ and the denominator $d$ have a one. We use zero denominator to represent $\pm \infty$.

If $I_{1}, \ldots, I_{d}$ denote index sets, tensors $u \in \mathbb{Q}^{I_{1} \times \cdots \times I_{d}}$ are represented by many-sorted structures, with one sort $\bar{I}$ for each index set $I$ on the list $I_{1}, \ldots, I_{d}$, and one sort $\bar{B}$ for a domain $\{0, \ldots, N\}$ of bit positions. The vocabulary $L_{\mathrm{vec}, d}$ of these structures has a binary relation symbol $\leq$ for the natural linear order on $\{0, \ldots, N\}$ and three $d+1$-ary relation symbols $P_{s}$, $P_{n}$ and $P_{d}$ for encoding the signs and the bits of the numerators and the denominators of the entries of the tensor. Matrices $\mathbf{A} \in \mathbb{Q}^{I \times J}$ and vectors $\mathbf{a} \in \mathbb{Q}^{I}$ are special cases of these. Indexed sets of vectors $\left\{\mathbf{a}_{i}: i \in K\right\} \subseteq \mathbb{Q}^{I}$ and index sets of rationals $\left\{b_{i}: i \in K\right\} \subseteq \mathbb{Q}$ too.

\subsection{Polytopes, lifts, and shadows}

A polytope is a set of the form $P=\left\{\mathbf{x} \in \mathbb{R}^{U}: \mathbf{A x} \leq \mathbf{b}\right\}$, where $U$ and $V$ are abstract non-empty index sets, $\mathbf{A} \in \mathbb{R}^{V \times U}$ is a constraint matrix, and $\mathbf{b} \in \mathbb{R}^{V}$ is an offset vector. If we think of $\mathbf{x}=\left(x_{u}\right)_{u \in U}$ as a sequence of variables, then the defining system of inequality constraints $\mathbf{A x} \leq \mathbf{b}$ is called a linear program (LP) for $P$. Note that the defining LPs for polytopes are by no means unique. Typically $\mathbf{A}$ and $\mathbf{b}$ can be chosen to have rational entries, in which case $P$ is represented by a sequence of linear constraints $\left(\gamma_{v}\right)_{v \in V}$ of one of its defining LPs with rational entries; i.e., each $\gamma_{v}$ is of the form $\mathbf{a}_{v}^{\mathrm{T}} \mathbf{x} \leq b_{v}$, with $\mathbf{a}_{v} \in \mathbb{Q}^{U}$ and $b_{v} \in \mathbb{Q}$. The size of such an LP is $(|U|+1)|V| b$, where $b$ is the maximum number of bits it takes to write all the numerators and all the denominators of the entries of the $\mathbf{a}_{v}$ and $b_{v}$ in binary.

If $\mathbf{x} \in \mathbb{R}^{U}$ and $\mathbf{y}_{1}, \ldots, \mathbf{y}_{m} \in \mathbb{R}^{U}$, and $\alpha_{1}, \ldots, \alpha_{m} \in \mathbb{R}$ are such that $\mathbf{x}=\sum_{i=1}^{m} \alpha_{i} \mathbf{y}_{i}$, with $\alpha_{i} \geq 0$ and $\sum_{i=1}^{m} \alpha_{i}=1$, then we say that $\mathbf{x}$ is a convex combination of $\mathbf{y}_{1}, \ldots, \mathbf{y}_{m}$. When $0<\alpha_{i}<1$ for some $i \in[m]$, the convex combination is called non-trivial. The convex hull $\operatorname{conv}\left(\mathbf{y}_{1}, \ldots, \mathbf{y}_{m}\right)$ is the set of all convex combinations of $\mathbf{y}_{1}, \ldots, \mathbf{y}_{m}$. A point $\mathbf{x}$ of a polytope $P$ is called a vertex if it cannot be expressed as a non-trivial convex combination of any two other points of $P$. If $P$ is a polytope and $P \subseteq\left\{\mathbf{x} \in \mathbb{R}^{U}: \mathbf{a}^{\mathrm{T}} \mathbf{x} \leq b\right\}$, then the set $\left\{\mathbf{x} \in P: \mathbf{a}^{\mathrm{T}} \mathbf{x}=b\right\}$ is called face of $P$. The faces of dimension 0 are the vertices of $P$; the faces of dimension 1 are called edges; the faces of dimension $\operatorname{dim}(P)-1$ are called facets, where $\operatorname{dim}(P)$ is the dimension of $P$. Each polytope has finitely many faces of each 
dimension; in particular finitely many vertices (see [36]). A polytope is bounded if and only if it is the convex hull of its vertices.

If $P \subseteq \mathbb{R}^{U} \times \mathbb{R}^{W}$ is a polytope, its projection into $\mathbb{R}^{U}$ is the set of points $\mathbf{x} \in \mathbb{R}^{U}$ for which there exists a point $\mathbf{y} \in \mathbb{R}^{W}$ with $(\mathbf{x}, \mathbf{y}) \in P$. The projection of a polytope is again a polytope. If $Q$ is the projection of $P$ into $\mathbb{R}^{U}$, then we say that $Q$ is a shadow of $P$, and that $P$ is a lift of $Q$. If $A, B \subseteq\{0,1\}^{U}$ are disjoint, then we say that $Q$ is a polytope that separates $A$ from $B$ if $A \subseteq Q$ and $B \subseteq \mathbb{R}^{U} \backslash Q$. We also say that $P$ is a polytope lift that separates $A$ from $B$. If $Q$ separates $A$ from its complement $\bar{A}=\{0,1\}^{U} \backslash A$, then we say that $Q$ is a polytope that recognizes $A$, and that $P$ is a polytope lift that recognizes $A$. Since no point in $\{0,1\}^{U}$ is in the convex hull of any set of points in $\{0,1\}^{U}$ that does not contain it, the convex hull of $A \subseteq\{0,1\}^{U}$ always recognizes $A$. The converse is not true: a polytope could recognize $A$ and not be the convex hull of $A$.

Let $P \subseteq \mathbb{R}^{U}$ be given by a sequence of linear constraints $\left(\gamma_{v}\right)_{v \in V}$. If a group $G$ acts on the set $U$, then for any $\gamma_{v}$ of the form $\mathbf{a}_{v} \mathbf{x} \leq b_{v}$, we write $\gamma_{v}^{\pi}$ for the linear constraint $\mathbf{a}_{v} \mathbf{x}^{\pi} \leq b_{v}$. Note that the sequence $\left(\gamma_{v}^{\pi}\right)_{v \in V}$ defines $P^{\pi} \subseteq \mathbb{R}^{U}$, which is again a polytope. As long as this does not lead to confusion, we identify polytopes with sequences of linear constraints that represent them. In particular, if we assume the polytope $P \subseteq \mathbb{R}^{U}$ to be represented by the sequence of constraints $\left(\gamma_{v}\right)_{v \in V}$, then by $P^{\pi}$ we mean both the permuted polytope itself, and its representation by the sequence of constraints $\left(\gamma_{v}^{\pi}\right)_{v \in V}$.

Let $U$ be a $G$-set. A polytope $P \subseteq \mathbb{R}^{U} \times \mathbb{R}^{W}$ is said to be $G$-symmetric if for every $\pi \in G$ there exists a permutation $\sigma \in \operatorname{Sym}_{W}$ such that $P^{(\pi, \sigma)}=P$. If additionally we are given an action of the group $G$ on $W$ such that $P^{(\pi, \pi)}=P$, then we say that the polytope $P$ is $G$-symmetric with respect to this action. A pair of permutations $(\pi, \sigma) \in \operatorname{Sym}_{U} \times \operatorname{Sym}_{W}$ such that $P^{(\pi, \sigma)}=P$ is called an automorphism of $P$. Hence, if $G \leq \operatorname{Sym}_{U}$, the fact that the polytope $P$ is $G$-symmetric is equivalent to the possibility of extending every permutation $\pi \in G$ to an automorphism of $P$.

For any $n \in \mathbb{N}$, if the set $[n]^{2}$ comes with the natural action of the symmetric group Sym $_{n}$, then any Sym $_{n}$-symmetric polytope $P \subseteq \mathbb{R}^{[n]^{2}} \times \mathbb{R}^{W}$ is said to be graph-symmetric. It is not difficult to see that any set $A \subseteq\{0,1\}^{[n]^{2}}$ recognised by a graph-symmetric polytope lift $P \subseteq \mathbb{R}^{[n]^{2}} \times \mathbb{R}^{W}$ is invariant with respect to the action of the group $\mathrm{Sym}_{n}$, i.e., for any $\mathbf{a} \in A$ and any $\pi \in \operatorname{Sym}_{n}$, we have $\mathbf{a}^{\pi} \in A$. The polytope lift $P$ can be therefore seen as recognising a class of graphs with $n$-vertices. If we take a graph $G$ with the set of vertices $V$ of size $n$, fix a bijection $f$ from $[n]$ to $V$, and define a vector $\mathbf{a}=\left(a_{i j}\right)_{i, j \in[n]} \in\{0,1\}^{[n]^{2}}$ by: $a_{i j}=1$ if and only if there is an edge from $f(i)$ to $f(j)$ in $G$, then $G$ belongs to the class recognised by $P$ if and only if $\mathbf{a} \in A$. Since $A$ is a $\operatorname{Sym}_{n}$-set, this definition does not depend on the choice of $f$.

More generally, we consider $\mathrm{Sym}_{n}$-symmetric polytope lifts recognising properties of arbitrary $L$-structures. For any $n \in \mathbb{N}$ and any single-sorted vocabulary $L$, let $L(n)$ be the disjoint union of $[n]^{\operatorname{ar}(R)}$ over all relation symbols $R$ in $L$. Since $L(n)$ comes with the natural action of the group $\operatorname{Sym}(n)$, we can talk about $\operatorname{Sym}_{n}$-symmetric polytopes over $\mathbb{R}^{L(n)} \times \mathbb{R}^{W}$. Any such polytope is called $L$-symmetric. As a straightforward generalisation of the discussion in the previous paragraph, $L$-symmetric polytope lifts over $\mathbb{R}^{L(n)} \times \mathbb{R}^{W}$ are defined to 
recognise classes of $L$-structures with $n$-element domains.

\subsection{Boolean circuits}

A circuit with inputs $\left(x_{u}\right)_{u \in U}$ is a directed acyclic graph $G$ whose vertices of zero in-degree are labelled by some input $x_{u}$, and every other vertex is labelled by a function from some fixed basis of symmetric Boolean functions, with the constraint that the function takes the same number of inputs as the in-degree of the vertex. The vertices of zero out-degree are called outputs. If $\left(y_{v}\right)_{v \in V}$ is a fixed naming of the outputs, then a circuit computes a Boolean function $f:\{0,1\}^{U} \rightarrow\{0,1\}^{V}$ in the obvious way. When $m=1$ we say that it recognizes $f^{-1}(1)$. The vertices of a circuit are also called gates. The size of the circuit is its number of gates. A Boolean threshold circuit is one whose gates are labelled by NOTs, unbounded degree ANDs, unbounded degree ORs, or unbounded degree thresholds $\mathrm{TH}_{n, k}$, where $\mathrm{TH}_{n, k}\left(z_{1}, \ldots, z_{n}\right)$ outputs 1 if, and only if, the number of 1 's in the input $z_{1}, \ldots, z_{n}$ is at least $k$.

If $U$ is a $G$-set and $W$ denotes the set of gates of $C$, we say that $C$ is $G$-symmetric if for every $\pi \in G$ there exists $\sigma \in \operatorname{Sym}_{W}$ such that $C^{(\pi, \sigma)}=C$, where by $C^{(\pi, \sigma)}$ we mean that the gates of the circuit are permuted according to $\sigma$, the labels from $\left\{x_{u}\right\}_{u \in U}$ are permuted according to $\pi$, and none of the other labels is moved. A circuit with $U=L(n)$ is called $L$-symmetric if it is $\operatorname{Sym}_{n}$-symmetric, with the natural action of $\operatorname{Sym}_{n}$ on $L(n)$. As for polytopes, we consider $L$-symmetric circuits as recognizing classes of $L$-structures on abstract sets $V$ of vertices through bijections $f:[n] \rightarrow V$. In the case of graphs, for example, in which $L(n)=[n]^{2}$, we say that such a circuit accepts a graph $G$ with the set of vertices $V$ of size $n$ if for some, and hence every, bijection $f:[n] \rightarrow V$ it holds that $C(\mathbf{a})=1$, where $\mathbf{a}$ is the vector that describes the image of $G$ under $f^{-1}$, as in the previous section.

\section{From Circuits to LPs}

In this section we prove the half of the equivalence that takes symmetric circuits with threshold gates into symmetric LPs. That is:

Lemma 1. If $\mathscr{C}$ is a class of L-structures that is recognized by a family of L-symmetric Boolean threshold circuits of size $s(n)$, then $\mathscr{C}$ is recognized by a family of L-symmetric LP lifts of size $s(n)^{O(1)}$. In addition, if the Boolean circuits do not have threshold gates, then the size of the LP lifts is $O(s(n))$.

The main step in the construction is the simulation of the threshold gates. The naïve approach by which each threshold gate is replaced by an equivalent AND-OR-NOT circuit will not work: it is known that any symmetric such circuit that computes the majority function must have superpolynomial size. This follows from Theorem 2 in [3] and a standard Ehrenfeucht-Fraissé argument. We need an alternative approach. As a step towards our goal, first we need to generalize the so-called Parity Polytope from Yannakakis [40]. 


\subsection{The parity polytope explained}

Yannakakis gives a polynomial-size symmetric polytope lift of the parity polytope

$$
\operatorname{PP}(n):=\operatorname{conv}\left\{\left(x_{1}, \ldots, x_{n}\right) \in\{0,1\}^{n}: \sum_{k=1}^{n} x_{k} \equiv 1(\bmod 2)\right\} .
$$

Note that this polytope has the following interesting feature:

if $x_{1}, \ldots, x_{n-1}$ are in $\{0,1\}$, then there exists a unique $x_{n}$ in $\mathbb{R}$ such that $\left(x_{1}, \ldots, x_{n}\right)$ is in $\operatorname{PP}(n)$, and moreover this $x_{n}$ is the unique bit in $\{0,1\}$ that makes the total sum $\sum_{k=1}^{n} x_{k}$ odd.

For the existence just take $x_{n} \in\{0,1\}$ so that $\sum_{k=1}^{n} x_{k}$ is odd. The uniqueness will follow once we show that any $x_{n}$ for which the extension vector $\left(x_{1}, \ldots, x_{n}\right)$ belongs to $\operatorname{PP}(n)$ is in $\{0,1\}$. In turn, this follows from the fact that all extreme points of $\operatorname{PP}(n)$ are in $\{0,1\}^{n}$, all have the same parity, and a single bit-flip flips their parity. Indeed, if $\left(x_{1}, \ldots, x_{n}\right)$ is in $\operatorname{PP}(n)$ but is not an extreme point, then it must be a non-trivial convex combination of at least two extreme points and, whenever $x_{1}, \ldots, x_{n-1}$ are all in $\{0,1\}$, only two candidate extreme points remain: $\left(x_{1}, \ldots, x_{n-1}, 0\right)$ and $\left(x_{1}, \ldots, x_{n-1}, 1\right)$. Otherwise some $x_{i}$ with $1 \leq i \leq n-1$ would be strictly between 0 and 1 . However, among these two candidate extreme points, at least one does not have the right parity, and hence is not even in $\operatorname{PP}(n)$.

The construction of the lift of $\mathrm{PP}(n)$ relies on the fact that the convex hulls of the Hamming-weight slices of the $n$-dimensional Boolean hypercube are definable by a small linear program. Precisely, for each $t \in[n]$ let

$$
\operatorname{EX}(n, t):=\operatorname{conv}\left\{\left(x_{1}, \ldots, x_{n}\right) \in\{0,1\}^{n}: \sum_{k=1}^{n} x_{k}=t\right\} .
$$

Then it holds that the direct relaxation is tight:

$$
\operatorname{EX}(n, t)=\left\{\left(x_{1}, \ldots, x_{n}\right) \in \mathbb{R}^{n}: \sum_{k=1}^{n} x_{k}=t, 0 \leq x_{k} \leq 1 \text { for every } k=1, \ldots, n\right\} .
$$

We provide an $\pm \epsilon$-proof since it is instructive. Let $P$ be the polytope defined by the linear program in the right-hand side of (4). The inclusion $\operatorname{EX}(n, t) \subseteq P$ is obvious. For the inclusion $P \subseteq \operatorname{EX}(n, t)$ it suffices to show that every vertex of $P$ has integral components; i.e., that every point in $P$ that has some non-integral component fails to be a vertex of $P$ because it is a non-trivial convex combination of two other points in $P$. Let $\mathbf{x}=\left(x_{1}, \ldots, x_{n}\right)$ be a point in $P$, let $I$ be the set of indices $k \in[n]$ such that $0<x_{k}<1$, and assume that $I \neq \emptyset$. Now define

$$
\epsilon:=\min \left\{x_{k}: k \in I\right\} \cup\left\{1-x_{k}: k \in I\right\} .
$$

Thus $\epsilon>0$. Since $\sum_{k=1}^{n} x_{k}=t$ and $t$ is an integer, necessarily $|I| \geq 2$. Fix two different indices $i$ and $j$ in $I$ and, for $b \in\{0,1\}$, let $\mathbf{x}_{b}=\left(x_{b, 1}, \ldots, x_{b, n}\right)$ be the point defined by

$$
\begin{array}{ll}
x_{b, k}:=x_{k}+(-1)^{b} \epsilon & \text { for } k=i \\
x_{b, k}:=x_{k}-(-1)^{b} \epsilon & \text { for } k=j \\
x_{b, k}:=x_{k} & \text { for } k \in[n] \backslash\{i, j\} .
\end{array}
$$


By the choice of $\epsilon$ we have $0 \leq x_{b, k} \leq 1$ for every $k \in[n]$ and every $b \in\{0,1\}$. Also

$$
\sum_{k=1}^{n} x_{b, k}=\sum_{k=1}^{n} x_{k}+\epsilon-\epsilon=\sum_{k=1}^{n} x_{k}=t
$$

for both $b \in\{0,1\}$, and

$$
\frac{1}{2}\left(x_{0, k}+x_{1, k}\right)=\frac{1}{2}\left(x_{k}+x_{k}\right)=x_{k}
$$

for every $k \in[n]$. Since $\epsilon>0$, it follows that $\mathbf{x}_{0}$ and $\mathbf{x}_{1}$ are distinct points in $P$ such that $\mathbf{x}=\frac{1}{2}\left(\mathbf{x}_{0}+\mathbf{x}_{1}\right)$. Thus $\mathbf{x}$ is not a vertex of $P$.

With the polytope $\operatorname{EX}(n, t)$ in hand we are ready to describe the lift of $\operatorname{PP}(n)$. First note that a real vector $\mathbf{x} \in \mathbb{R}^{n}$ is in $\operatorname{PP}(n)$ if, and only if, $\mathbf{x}=\sum_{t} w_{t} \mathbf{y}_{t}$ where each vector $\mathbf{y}_{t}$ is in $\operatorname{EX}(n, 2 t+1)$, and the $w_{t}$ 's are non-negative coefficients that add up to one, with $t$ ranging over $\{0, \ldots,\lfloor n / 2\rfloor\}$. In order to express this as a linear program we introduce variables $w_{t}$ and $z_{t, i}$ for each $t \in\{0, \ldots,\lfloor n / 2\rfloor\}$ and $i \in\{1, \ldots, n\}$ with the intention that $z_{t, i}=w_{t} y_{t, i}$ for appropriate values $y_{t, i}$ that we do not care to actually get. Writing $T$ for $\{0, \ldots,\lfloor n / 2\rfloor\}$ and $N$ for $\{1, \ldots, n\}$, the linear program that achieves this is the following:

$$
\begin{array}{ll}
\sum_{t \in T} w_{t}=1 & \text { for each } t \in T \\
0 \leq w_{t} \leq 1 & \text { for each } i \in N \\
\sum_{t \in T} z_{t, i}=x_{i} & \text { for each } t \in T \\
\sum_{i \in N} z_{t, i}=(2 t+1) w_{t} & \text { for each } t \in T \text { and } i \in N \\
0 \leq z_{t, i} \leq w_{t} & \text { r }
\end{array}
$$

The symmetry of this linear program with respect to the $x$-variables is obvious: given a permutation $\pi \in \operatorname{Sym}_{n}$, let $\sigma$ be the permutation that maps $z_{t, i}$ to $z_{t, \pi(i)}$ and leaves each $w_{t}$ in place.

\subsection{The truncated parity polytope}

For each integer $n \geq 1$, let $|n|$ be the number of bits it takes to write $n$ in binary. We want lifts of the following truncated parity polytopes defined for each pair of integers $n \geq 1$ and $q \in\{0, \ldots,|n|-1\}$ :

$$
\mathrm{PP}(n, q):=\operatorname{conv}\left\{\left(x_{1}, \ldots, x_{n}\right) \in\{0,1\}^{n}:\left\lfloor 2^{-q} \sum_{k=1}^{n} x_{k}\right\rfloor \equiv 1(\bmod 2)\right\} .
$$

This time a vector $\mathbf{x} \in \mathbb{R}^{n}$ is in $\operatorname{PP}(n, q)$ if, and only if, $\mathbf{x}=\sum_{t, r} w_{t, r} \mathbf{y}_{t, r}$ where each vector $\mathbf{y}_{t, r}$ is in $\operatorname{EX}\left(n, 2^{q}(2 t+1)+r\right)$, and the $w_{t, r}$ are non-negative coefficients that add up to one, with $(t, r)$ ranging over the set of pairs of integers with $t \in\left\{0, \ldots,\left\lfloor n / 2^{q+1}\right\rfloor\right\}$ and $r \in\left\{0, \ldots, 2^{q}-1\right\}$. We introduce variables $w_{t, r}$ and $z_{t, r, i}$ for each $(t, r) \in T$ and each $i \in N$, where $T=\left\{0, \ldots,\left\lfloor n / 2^{q+1}\right\rfloor\right\} \times\left\{0, \ldots, 2^{q}-1\right\}$ and $N=\{1, \ldots, n\}$, with the intention that $z_{t, r, i}=w_{t, r} y_{t, r, i}$ for appropriate values $y_{t, r, i}$ that we do not care to actually get. The linear 
program that achieves this is the following:

$$
\begin{array}{ll}
\sum_{(t, r) \in T} w_{t, r}=1 & \text { for each }(t, r) \in T \\
0 \leq w_{t, r} \leq 1 & \text { for each } i \in N \\
\sum_{(t, r) \in T} z_{t, r, i}=x_{i} & \text { for each }(t, r) \in T \\
\sum_{i \in N} z_{t, r, i}=\left(2^{q}(2 t+1)+r\right) w_{t, r} & \text { for each } t \in T \text { and } i \in N \\
0 \leq z_{t, r, i} \leq w_{t, r} &
\end{array}
$$

The symmetry of this linear program with respect to the $x$-variables is again obvious: given $\pi \in \mathrm{Sym}_{n}$, let $\sigma$ map $z_{t, r, i}$ to $z_{t, r, \pi(i)}$ and leave every other variable in place. The polytope $\operatorname{PP}(n, q)$ has the following interesting feature that is analogous to the one we argued for $\operatorname{PP}(n)$ :

if $x_{1}, \ldots, x_{n-1}$ are in $\{0,1\}$ and $\sum_{k=1}^{n-1} x_{k} \equiv-1\left(\bmod 2^{q}\right)$, then there exists a unique $x_{n}$ in $\mathbb{R}$ such that $\left(x_{1}, \ldots, x_{n}\right)$ is in $\operatorname{PP}(n, q)$, and moreover $x_{n}$ is the unique bit in $\{0,1\}$ that makes the truncation $\left\lfloor 2^{-q} \sum_{k=1}^{n} x_{k}\right\rfloor$ odd.

For the existence just take $x_{n} \in\{0,1\}$ so that the truncation $\left\lfloor 2^{-q} \sum_{k=1}^{n} x_{k}\right\rfloor$ is odd, which must exist by the assumption that $\sum_{k=1}^{n-1} x_{k} \equiv-1\left(\bmod 2^{q}\right)$. The uniqueness follows once we show that every $x_{n}$ for which the extension vector $\left(x_{1}, \ldots, x_{n}\right)$ belongs to $\operatorname{PP}(n, q)$ is in $\{0,1\}$, and again the assumption that $\sum_{k=1}^{n-1} x_{k} \equiv-1\left(\bmod 2^{q}\right)$. For a proof that such an $x_{n}$ is in $\{0,1\}$ it suffices to show that if $\left(x_{1}, \ldots, x_{n}\right) \in \operatorname{PP}(n, q)$ satisfies the conditions, then it is an extreme point. If it were not an extreme point then it would be a non-trivial combination of at least two extreme points and, whenever $x_{1}, \ldots, x_{n-1}$ are all in $\{0,1\}$, only two candidates remain: $\left(x_{1}, \ldots, x_{n-1}, 0\right)$ and $\left(x_{1}, \ldots, x_{n-1}, 1\right)$; otherwise some $x_{i}$ with $1 \leq i \leq n-1$ would be strictly between 0 and 1 . However, assuming that $\sum_{k=1}^{n-1} x_{k} \equiv-1\left(\bmod 2^{q}\right)$, at least one of these extreme points must have even truncation $\left\lfloor 2^{-q} \sum_{k=1}^{n} x_{k}\right\rfloor$, and hence not even belong to $\operatorname{PP}(n, q)$; a contradiction.

\subsection{Counting gates}

The goal in this subsection is to write a polynomial-size symmetric linear program that can be used to simulate exact counting gates $\operatorname{EX}_{n, t}\left(x_{1}, \ldots, x_{n}\right)$, which outputs 1 if the sum of the $n$ input bits is exactly $t$, and 0 otherwise. In order to do this we use the truncated parity polytopes to compute the bits of the binary representation of $\sum_{k=1}^{n} x_{i}$, and then compare the result with the bits of the binary representation of $t$.

First consider the following sequence of linear programs which depend only on $n$ and 
not on $t$ :

$$
\begin{aligned}
& \left(x_{1}, \ldots, x_{n}, 1,1-z_{1}\right) \in \operatorname{PP}(n+2,0) \\
& \left(x_{1}, \ldots, x_{n}, 1,1, z_{1}, 1-z_{2}\right) \in \operatorname{PP}(n+4,1) \\
& \left(x_{1}, \ldots, x_{n}, 1,1,1,1, z_{1}, z_{2}, z_{2}, 1-z_{3}\right) \in \operatorname{PP}(n+8,2) \\
& \vdots \\
& \left(x_{1}, \ldots, x_{n}, 1^{\left(2^{q}\right)}, z_{1}^{(1)}, z_{2}^{(2)}, \ldots, z_{q}^{\left(2^{q-1}\right)}, 1-z_{q+1}\right) \in \operatorname{PP}\left(n+2^{q+1}, q\right) \\
& \vdots \\
& \left(x_{1}, \ldots, x_{n}, 1^{\left(2^{|n|-1}\right)}, z_{1}^{(1)}, z_{2}^{(2)}, \ldots, z_{|n|-1}^{\left(2^{|n|-2}\right)}, 1-z_{|n|}\right) \in \operatorname{PP}\left(n+2^{|n|},|n|-1\right),
\end{aligned}
$$

where, for $\ell \geq 1$, the notation $a^{(\ell)}$ denotes the string $a, a, \ldots, a$ of length $\ell$. We claim the following property:

if $x_{1}, \ldots, x_{n}$ are in $\{0,1\}$, then there is a unique vector $\left(z_{1}, \ldots, z_{|n|}\right) \in \mathbb{R}^{|n|}$ which together with $x_{1}, \ldots, x_{n}$ is a solution, and in this solution $z_{k} \in\{0,1\}$ for all $k$, and $\sum_{k=1}^{n} x_{k}=\sum_{k=1}^{|n|}\left(1-z_{k}\right) 2^{k-1}$; in other words, $z_{1}, \ldots, z_{|n|}$ are the flips of the bits of the binary representation of $\sum_{k=1}^{n} x_{k}$, listed from least to most significant bit.

From now on in this proof, let $X=\sum_{k=1}^{n} x_{k}$. The first part of the claim follows from the corresponding property of $\operatorname{PP}(n, q)$ 's, and induction on $q=0,1, \ldots,|n|-1$. The second part is proved by showing that $z_{1}, \ldots, z_{q+1}$ are all in $\{0,1\}$ and

$$
X \equiv \sum_{k=1}^{q+1}\left(1-z_{k}\right) 2^{k-1}\left(\bmod 2^{q+1}\right)
$$

also by induction on $q=0,1, \ldots,|n|-1$. For $q=0$ the claim is true since, by the main property of $\operatorname{PP}(n+2,0)=\operatorname{PP}(n+2)$, there is a unique $z_{1}$ for which $\left(x_{1}, \ldots, x_{n}, 1,1-z_{1}\right)$ is in $\operatorname{PP}(n+2)$, and this is the unique bit that makes $X+1+1-z_{1}$ odd, hence $X-z_{1}$ odd. Assume now that $z_{1}, \ldots, z_{q+1}$ are all in $\{0,1\}$ and that (14) holds for $q \in\{0, \ldots,|n|-2\}$ and we prove it for $q+1$. First observe that

$$
X+2^{q+1}+\sum_{k=1}^{q+1} z_{k} 2^{k-1} \equiv X+\sum_{k=1}^{q+1} z_{k} 2^{k-1} \equiv 2^{q+1}-1 \equiv-1\left(\bmod 2^{q+1}\right),
$$

where in the second equivalence we used the induction hypothesis on $q$. Moreover $z_{1}, \ldots, z_{q+1}$ are all in $\{0,1\}$, also by induction hypothesis on $q$. Thus, the vector

$$
\left(x_{1}, \ldots, x_{n}, 1^{\left(2^{q+1}\right)}, z_{1}^{(1)}, z_{2}^{(2)}, \ldots, z_{q+1}^{\left(2^{q+1-1}\right)}, 1-z_{q+2}\right)
$$

satisfies the hypothesis of property (13) for $\operatorname{PP}\left(n+2^{q+2}, q+1\right)$. It follows that there is a unique $z_{q+2}$ that puts (16) in $\operatorname{PP}\left(n+2^{q+2}, q+1\right)$, and this is the unique bit such that the quantity

$$
\left\lfloor 2^{-(q+1)}\left(X+2^{q+1}+\sum_{k=1}^{q+1} z_{k} 2^{k-1}+1-z_{q+2}\right)\right\rfloor
$$


is odd. Now, (14) says that

$$
X=2^{q+1}\left\lfloor 2^{-(q+1)} X\right\rfloor+\sum_{k=1}^{q+1}\left(1-z_{k}\right) 2^{k-1},
$$

which means that the unique bit that makes the quantity in (17) odd is the flip of the $(q+1)$-th least significant bit of $X$.

Now, exact- $t$ counting gates can be expressed using an additional linear program that simulates an AND gate to compare the bits $z_{1}, \ldots, z_{|n|}$ with (the flips of) the bits of the binary representation of $t$. For both $b \in\{0,1\}$, let $K_{b} \subseteq[|n|]$ be the subset of bit-positions at which the $|n|$-long binary representation of $t$ is $b$. Then, the relation $y=\operatorname{EX}_{n, t}\left(x_{1}, \ldots, x_{n}\right)$ is expressed by the union of (12) and the following:

$$
\begin{array}{ll}
y \geq \sum_{k \in K_{0}} z_{k}+\sum_{k \in K_{1}}\left(1-z_{k}\right)-|n|+1 & \\
y \leq z_{k} & \text { for every } k \in K_{0} \\
y \leq 1-z_{k} & \text { for every } k \in K_{1} \\
0 \leq y \leq 1 . &
\end{array}
$$

We write $\operatorname{EX}_{n, t}\left(x_{1}, \ldots, x_{n}, y\right)$ to denote the LP that has all the constraints in (12) and all the constraints in (19). We summarize its main properties in the following:

Lemma 2. The linear program $\operatorname{EX}_{n, t}\left(x_{1}, \ldots, x_{n}, y\right)$ has size polynomial in $n$, is symmetric with respect to the group of permutations of $x_{1}, \ldots, x_{n}, y$ that fix $y$, and has the following property: If $x_{1}, \ldots, x_{n}$ are all in $\{0,1\}$, then there is a unique $y \in \mathbb{R}$ such that $\left(x_{1}, \ldots, x_{n}, y\right)$ can be extended to a feasible solution, and this $y$ is the unique output bit of the corresponding gate evaluated on inputs $x_{1}, \ldots, x_{n}$.

Proof. The bound on the size follows by inspection. The symmetry with respect to the group of permutations of $x_{1}, \ldots, x_{n}, y$ that fix $y$ follows from the symmetry claims for the truncated parity polytopes, together with the extension that keeps each $z_{i}$-variable in place. For proving the main property, assume that $x_{1}, \ldots, x_{n}$ are all in $\{0,1\}$. The first part (12) of $\operatorname{EX}_{n, t}\left(x_{1}, \ldots, x_{n}, y\right)$ has the feature expressed in property (13). Let then $z_{1}, \ldots, z_{|n|}$ satisfy the conclusion in that property. Thus, $z_{1}, \ldots, z_{|n|}$ are the flips of the bits of the binary representation of $\sum_{i=1}^{n} x_{i}$. In particular they are all in $\{0,1\}$. The second part (19) of $\operatorname{EX}_{n, t}\left(x_{1}, \ldots, x_{n}, y\right)$ has the feature that if all $z_{1}, \ldots, z_{|n|}$ are in $\{0,1\}$, then there is a unique $y$ in $\mathbb{R}$ that makes a solution, and this $y$ is precisely the bit that is 1 if and only if all $z_{k}$ with $k \in K_{0}$ are one, and all $z_{k}$ with $k \in K_{1}$ are zero. In other words, $y=1$ if and only if $z_{1}, \ldots, z_{|n|}$ are the flips of the bits of the binary representation of $t$, and hence if and only if $\sum_{k=1}^{n} x_{k}=t$. 


\subsection{The construction}

Let us recall how AND, OR and NOT gates are represented by LPs. The LPs for these three types of gates do not require auxiliary variables, and their size is linear in the fan-in. Define:

$$
\begin{array}{lll}
\frac{\operatorname{AND}\left(x_{1}, \ldots, x_{n}, y\right)}{y \geq \sum_{i=1}^{n} x_{i}-n+1} & \frac{\operatorname{OR}\left(x_{1}, \ldots, x_{n}, y\right)}{1-y \geq \sum_{i=1}^{n}\left(1-x_{i}\right)-n+1} & \frac{\operatorname{NOT}(x, y)}{y=1-x} \\
y \leq x_{i} & 1-y \leq 1-x_{i} & 0 \leq x \leq 1 \\
0 \leq x_{i} \leq 1 & 0 \leq x_{i} \leq 1 & 0 \leq y \leq 1 . \\
0 \leq y \leq 1 . & 0 \leq y \leq 1 . &
\end{array}
$$

The main properties of these LPs are summarized in the following:

Lemma 3. The linear programs $\operatorname{AND}\left(x_{1}, \ldots, x_{n}, y\right), \mathrm{OR}\left(x_{1}, \ldots, x_{n}, y\right)$, and $\operatorname{NOT}\left(x_{1}, y\right)$ have size linear in $n$, are symmetric with respect to the group of permutations of its variables that fix $y$, and have the following property: If $x_{1}, \ldots, x_{n}$ are all in $\{0,1\}$, with $n=1$ for NOT, then there is a unique $y \in \mathbb{R}$ that makes $\left(x_{1}, \ldots, x_{n}, y\right)$ feasible, and this $y$ is the unique output bit of the corresponding gate evaluated on inputs $x_{1}, \ldots, x_{n}$.

Proof. For NOT this is totally obvious. For AND it is easy to check, and for OR it follows from the corresponding properties of AND and NOT.

We define the conversion from circuits to LPs. Let $U$ be a set and let $C$ be a circuit with inputs $\left(x_{u}\right)_{u \in U}$. Let $C^{\prime}$ be the circuit that results from replacing each $k$-threshold gate with inputs $y_{1}, \ldots, y_{m}$ by $\bigvee_{t=k}^{m} \operatorname{EX}_{m, t}\left(y_{1}, \ldots, y_{m}\right)$, where $\operatorname{EX}_{m, t}\left(y_{1}, \ldots, y_{m}\right)$ denotes an exact counting gate with inputs $y_{1}, \ldots, y_{m}$ which outputs 1 if and only if the exact number of 1 's in the input is $t$. The resulting circuit is equivalent to $C$ and its number of gates is polynomial in that of $C$. We define the LP; we call it $\operatorname{LP}(C)$.

For each gate $i$ in $C^{\prime}$, let $y_{i}$ be a variable constrained by the inequalities

$$
0 \leq y_{i} \leq 1
$$

For each gate $o$ in $C^{\prime}$ add the constraints and the auxiliary variables, when necessary, that express their computation:

$$
\begin{array}{ll}
y_{o}=x_{u} & \text { if } o \text { is an input gate labelled by } x_{u}, \\
\operatorname{NOT}\left(y_{i}, y_{o}\right) & \text { if } o \text { is a NOT gate with input } i, \\
\operatorname{AND}\left(y_{i_{1}}, \ldots, y_{i_{m}}, y_{o}\right) & \text { if } o \text { is an AND gate with inputs } i_{1}, \ldots, i_{m}, \\
\operatorname{OR}\left(y_{i_{1}}, \ldots, y_{i_{m}}, y_{o}\right) & \text { if } o \text { is an OR gate with inputs } i_{1}, \ldots, i_{m}, \\
\operatorname{EX}_{m, t}\left(y_{i_{1}}, \ldots, y_{i_{m}}, y_{o}\right) & \text { if } o \text { is an EX } \operatorname{EX}_{m, t} \text { gate with inputs } i_{1}, \ldots, i_{m}, \\
y_{o}=1 & \text { if } o \text { is the output gate of the circuit. }
\end{array}
$$

By Lemmas 2 and 3, all six cases have size polynomial in the number of inputs, hence the total size is polynomial in the size of $C^{\prime}$. In case $C$ does not have TH gates, the step for replacing them by EX gates is not done, and all gates are AND, OR, NOT, so the size of the LP stays linear in the size of $C$. 
Lemma 4. If $U$ is a $G$-set and $C$ is $G$-symmetric, then $\operatorname{LP}(C)$ is $G$-symmetric and recognizes the same subset of $\{0,1\}^{U}$ as $C$.

Proof. The claim that $\operatorname{LP}(C)$ recognizes the same subset of $\{0,1\}^{U}$ as $C$ follows from Lemmas 2 and 3 . We prove the symmetry. First note that the intermediate circuit $C^{\prime}$ is also $G$-symmetric. Now we show that the LP is also $G$-symmetric. Fix some $\pi \in G$. Let $\sigma$ be a permutation of the gates of $C^{\prime}$ so that the pair $(\pi, \sigma)$ leaves $C^{\prime}$ in place. In particular, for each gate $o$ of $C^{\prime}$ with inputs $i_{1}, \ldots, i_{m}$, if $p=\sigma(o)$, then $p$ is the same type of gate as $o$, has the same fan-in $m$, and if $o$ is an input gate fed by $x_{u}$, then $p$ is an input gate fed by $x_{\pi(u)}$. Moreover, if $j_{1}, \ldots, j_{m}$ are the inputs of gate $p$, then there is a permutation $\tau_{o} \in \operatorname{Sym}_{m}$ so that $\sigma\left(i_{k}\right)=j_{\tau_{o}(k)}$ for every $k \in[\mathrm{m}]$. If we think of $\sigma$ as mapping the output variable $y_{o}$ of the linear program $P_{o}=P\left(y_{i_{1}}, \ldots, y_{i_{m}}, y_{o}\right)$ for gate $o$ to the output variable $y_{p}$ of the linear program $P_{p}=P\left(y_{j_{1}}, \ldots, y_{j_{m}}, y_{p}\right)$ for gate $p$, then, by the above, this map takes the input variables of $P_{o}$ to the input variables of $P_{p}$. We want to show that this $\sigma$ can be extended to also map the auxiliary variables of $P_{o}$ to the auxiliary variables of $P_{p}$ in such a way that the resulting extension of $\pi$ is an automorphism of the linear program $P$ for $C^{\prime}$. We define this extension automorphism gate by gate.

We start with the internal gates of $C^{\prime}$. By the symmetry claims in Lemmas 2 and 3 , the permutation that agrees with $\tau_{o}$ on the input variables $y_{j_{1}}, \ldots, y_{j_{m}}$ of $P_{p}$ and that fixes the output variable $y_{p}$, extends to an automorphism $\rho_{o}$ of $P_{p}$. With the automorphisms $\rho_{o}$ in hand, we are ready to define the automorphism of $P$ that extends $\pi$ : for each gate $o$, map the variable $y_{o}$ to $y_{\sigma(o)}$, and map each auxiliary variable of $P_{o}$ to the image under $\rho_{o}$ of the corresponding auxiliary variable of $P_{p}$, where $p=\sigma(o)$. For all internal gates, this has the required properties by construction. For the gates $o$ that are fed by a variable $x_{u}$, the gate $\sigma(o)$ must be fed by the variable $x_{\pi(u)}$, and therefore $y_{o}=x_{u}$ gets mapped to $y_{\sigma(o)}=$ $x_{\pi(u)}$, as required. For the output gate $o$ of $C^{\prime}$ we have $\sigma(o)=o$, and the constraint $y_{o}=1$ gets mapped to itself.

Proof of Lemma 1. This is a consequence of Lemma 4: for the $n$-th LP we let $U=L(n)$, let $G=\operatorname{Sym}_{n}$ with the natural action on $L(n)$, and use $\operatorname{LP}\left(C_{n}\right)$, where $C_{n}$ is the $n$-th circuit.

\section{From LPs to Logic}

We say that a function $s(n)$ is at most weakly exponential if there exists a positive real $\epsilon$ such that $s(n) \leq 2^{n^{1-\epsilon}}$ for every sufficiently large $n$. In this section we prove the second half of the equivalence which takes families of symmetric linear programs to families of formulas of counting logic. That is:

Lemma 5. If $\mathscr{C}$ is a class of L-structures that is recognized by a family of L-symmetric polytope lifts of size $s(n)$, then $\mathscr{C}$ is recognized by a family of $\mathrm{C}^{k(n)}$ formulas, where $k(n)=$ $O(\log (s(n)) /(\log (n)-\log \log (s(n))))$. Moreover, if $s(n)$ is at most weakly exponential, then the formulas have size $s(n)^{O(1)}$. 
The key technical tool is the notion of a bounded support. The existence of bounded supports implies that a property of $L$-structures recognised by a family of $L$-symmetric LP lifts is recognised by a family of manageable such LP lifts. Let us illustrate the notion of a manageable linear program by an example.

Consider a graph-symmetric polytope $P$ over $\mathbb{R}^{[2]^{2}} \times \mathbb{R}^{2}$ given by the following system of linear constraints:

$$
\begin{aligned}
& x_{11} \leq 1 \\
& x_{22} \leq 1 \\
& x_{12}-y_{12} \leq 0 \\
& x_{21}-y_{21} \leq 0 \\
& y_{12}+y_{21} \leq 1
\end{aligned}
$$

Two properties of $P$ are central to the notion of a manageable polytope. Firstly, each of the auxiliary variables $y_{12}, y_{21}$ is essentially a tuple of integers from [2] of a bounded length. Secondly, $P$ is graph-symmetric with respect to the natural action of the group $\mathrm{Sym}_{2}$ on the set $\left\{y_{12}, y_{21}\right\}$. Indeed, for any permutation $\pi \in \mathrm{Sym}_{2}$ the system of constraints:

$$
\begin{aligned}
& x_{\pi(1) \pi(1)} \leq 1 \\
& x_{\pi(2) \pi(2)} \leq 1 \\
& x_{\pi(1) \pi(2)}-y_{\pi(1) \pi(2)} \leq 0 \\
& x_{\pi(2) \pi(1)}-y_{\pi(2) \pi(1)} \leq 0 \\
& y_{\pi(1) \pi(2)}+y_{\pi(2) \pi(1)} \leq 1
\end{aligned}
$$

defines the same polytope. A formal definition of a manageable polytope is deferred to Subsection 4.3 ,

Given an $L$-structure over an $n$-element domain, a manageable polytope lift over $\mathbb{R}^{L(n)} \times$ $\mathbb{R}^{W}$ can be turned into a linear program whose variables are indexed by tuples of elements of the structure of bounded length. Moreover, the symmetry condition guarantees that this can be done without referring to any concrete bijection between $[n]$ and the domain of the structure. Thus, as we show, it can be performed by means of logical interpretations.

In what follows, from a family $\left(P_{n}\right)_{n \in \mathbb{N}}$ of $L$-symmetric LP lifts we obtain a family $\left(\bar{P}_{n}\right)_{n \in \mathbb{N}}$ of manageable LP lifts recognising the same family of structures. Lemma 6 below implies that from each $P_{n}$ one can construct a polytope lift $\widehat{P}_{n}$ which recognises the same subset of $\{0,1\}^{L(n)}$ but comes with an action of the group $\mathrm{Sym}_{n}$ witnessing its symmetry. Further, in Subsection 4.2 we show that the action of $\mathrm{Sym}_{n}$ on each of the constraints and auxiliary variables of $\widehat{P}_{n}$ depends on a subset of $[n]$ of bounded size called its support. In the second part of Subsection 4.2 we analyse properties of sets whose elements have bounded supports in order to show that they are essentially sets of tuples of integers from $[n]$. This implies, in Subsection 4.3, that each $\widehat{P}_{n}$ after a small modification becomes a manageable LP lift $\bar{P}_{n}$. Finally, in Subsection 4.4 based on $\bar{P}_{n}$ we construct a FOC-interpretation that given an $L$ structure $\mathbb{A}$ over an $n$-element domain outputs a linear program which has a solution if and 
only if $\mathbb{A}$ belongs to the class of interest (for a definition of an interpretation see e.g. [24]). Since solving linear programs is expressible in FPC [4], we are able to conclude the proof.

\subsection{Rigid polytopes}

In this subsection we consider general $G$-symmetric LPs, i.e., not necessarily $L$-symmetric.

Let $U$ be a $G$-set and let $P \subseteq \mathbb{R}^{U} \times \mathbb{R}^{W}$ be a $G$-symmetric polytope given by a sequence of linear constraints $\left(\gamma_{v}\right)_{v \in V}$ where each $\gamma_{v}$ is of the form $\mathbf{a}^{\mathrm{T}} \mathbf{x}+\mathbf{b}^{\mathrm{T}} \mathbf{y} \leq c$, with $\mathbf{x}=\left(x_{u}\right)_{u \in U}$ and $\mathbf{y}=\left(y_{w}\right)_{w \in W}$. We say that the polytope $P$ is rigid if for every $\pi \in G$ there exists a unique element of $\operatorname{Sym}_{W}$, let us denote it by $\sigma_{\pi}$, such that $P^{\left(\pi, \sigma_{\pi}\right)}=P$.

Assume that $P$ is rigid. It is easy see that the mapping from $G$ to $\operatorname{Sym}_{W}$ given by $\pi \mapsto \sigma_{\pi}$ is a group homomorphism. Hence, there is a natural action of the group $G$ on the set of auxiliary variables $\left\{y_{w}\right\}_{w \in W}$ such that for any $\pi \in G$ and $w \in W$ applying $\pi$ to $y_{w}$ gives $y_{\sigma_{\pi}(w)}$, and the polytope $P$ is $G$-symmetric with respect to this action. Moreover, this induces an action of the group $G$ on the set of linear constraints $\left\{\gamma_{v}\right\}_{v \in V}$ in the obvious way: for any $\pi \in G$ and any $v \in V$ applying $\pi$ to $\gamma_{v}$ of the form $\mathbf{a}^{\mathrm{T}} \mathbf{x}+\mathbf{b}^{\mathrm{T}} \mathbf{y} \leq c$ gives $\mathbf{a}^{\mathrm{T}} \mathbf{x}^{\pi}+\mathbf{b}^{\mathrm{T}} \mathbf{y}^{\sigma_{\pi}} \leq c$, and the symmetry of $P$ guarantees that this is also a constraint. For rigid $G$-symmetric polytopes, we write $\mathbf{y}^{\pi}$ to mean $\mathbf{y}^{\sigma_{\pi}}$, we use $\gamma_{v}^{\pi}$ to denote $\mathbf{a}^{\mathrm{T}} \mathbf{x}^{\pi}+\mathbf{b}^{\mathrm{T}} \mathbf{y}^{\sigma_{\pi}} \leq c$, and $P^{\pi}$ to denote $P^{\left(\pi, \sigma_{\pi}\right)}$.

Suppose that a subset $A$ of $\{0,1\}^{U}$ is recognised by a $G$-symmetric polytope lift $P$. We show that there exists a rigid $G$-symmetric polytope lift $\widehat{P}$ of size polynomial in the size of $P$ recognising $A$. More precisely, the number of auxiliary variables and the number of constraints of $\widehat{P}$ are at most, respectively, the number of auxiliary variables and the number of constraints of $P$, and the bit-size of the coefficients which appear in the linear constraints defining $\widehat{P}$ is polynomial in the bit-size of the coefficients which appear in the linear constraints defining $P$.

The construction of $\widehat{P}$ goes as follows. For the subgroup of $\operatorname{Sym}_{W}$ consisting of all permutations $\sigma$ such that $P^{(\mathrm{id}, \sigma)}=P$, consider the orbits of the set of auxiliary variables $\left\{y_{w}\right\}_{w \in W}$ under the action of this subgroup. By identifying the variables in each of those orbits we obtain a new $G$-symmetric polytope lift recognising $A$ with potentially smaller number of auxiliary variables. This procedure needs to be iterated until the obtained polytope is rigid. Let us provide more details.

We need a few pieces of notation. For every $\pi \in G$, by $\operatorname{ext}(\pi)$ we denote the set of all $\sigma \in \operatorname{Sym}_{W}$ satisfying $P^{(\pi, \sigma)}=P$ and by $H$ we denote the union of $\operatorname{ext}(\pi)$ over all the elements $\pi$ of $G$, i.e., $H=\left\{\sigma \in \operatorname{Sym}_{W}\right.$ : there exists $\pi \in G$ such that $\left.P^{(\pi, \sigma)}=P\right\}$. It is easy to see that both ext(id) and $H$ are subgroups of $\operatorname{Sym}_{W}$. Moreover, for any $\pi \in G$ the set $\operatorname{ext}(\pi)$ is a coset of $\operatorname{ext}(\mathrm{id})$ in $H$. Now let $K=\sum_{\pi \in G}\{\pi\} \times \operatorname{ext}(\pi)$. Observe that $K$ is a subgroup of the direct product $G \times H$ and consider the projection homomorphism $f_{G}: K \rightarrow G$ given by $f_{G}(\pi, \sigma)=\pi$. The kernel of this homomorphism is the group $\{$ id $\} \times \operatorname{ext}(\mathrm{id})$. Let us denote it by $J$. Since the homomorphism $f_{G}$ is surjective, the quotient $K / J$ is isomorphic to $G$.

Notice that the polytope $P$ is rigid if, and only if, the group ext(id) is trivial. Assume therefore that this is not the case and let $\mathcal{O}$ denote the set of orbits of the set of auxiliary 
variables $\left\{y_{w}\right\}_{w \in W}$ under the action of ext(id). Recall that $\mathcal{O}$ forms a partition of $\left\{y_{w}\right\}_{w \in W}$. For every orbit $O \in \mathcal{O}$, we introduce a new variable $y_{O}$ and for any polytope $R$, let $\widehat{R}$ denote the polytope obtained from $R$ by substituting, for each $O \in \mathcal{O}$, every variable in $O$ (if present) by $y_{O}$. We aim to show that $\widehat{P}$ is a $G$-symmetric polytope recognizing $A$.

The projection homomorphism $f_{H}: K \rightarrow H$ given by $f_{H}(\pi, \sigma)=\sigma$ can be seen as a homomorphism from $K$ to $\operatorname{Sym}_{W}$ and hence defines an action of the group $K$ on the set of variables $\left\{y_{w}\right\}_{w \in W}$. Since $J$ is a normal subgroup of $K$, the quotient group $K / J$ acts on the set of orbits of $\left\{y_{w}\right\}_{w \in W}$ under the (induced) action of $J$ : for any $\pi \in G, \sigma \in \operatorname{ext}(\pi)$ and $w \in W$, applying $(\pi, \sigma) J$ to the orbit of $y_{w}$ maps it to the orbit of $y_{\sigma(w)}$. Since the groups $J$ and ext(id) as well as the groups $K / J$ and $G$ are isomorphic, this gives us an action of $G$ on $\mathcal{O}$ which, as we argue below, witnesses the fact that the polytope $\widehat{P}$ is $G$-symmetric. Unfolding the abstract definition of this group action, there is a homomorphism $h$ from the group $G$ to the symmetric group $\operatorname{Sym}_{\mathcal{O}}$ such that for every $\pi \in G, h(\pi)$ is the permutation of $\mathcal{O}$, which for any $w \in W$, maps the orbit of the variable $y_{w}$ to the orbit of the variable $y_{\sigma(w)}$, where $\sigma$ is any permutation from $\operatorname{ext}(\pi)$. Finally, let us observe that, for any $\pi \in G$ and $\sigma \in \operatorname{ext}(\pi)$, it holds that $\widehat{P}^{(\pi, h(\pi))}=\widehat{P^{(\pi, \sigma)}}=\widehat{P}$, which means that indeed $\widehat{P}$ is $G$-symmetric. It remains to show that $\widehat{P}$ recognises $A$.

Let $\mathbf{x} \in A$ and take some $\mathbf{y} \in \mathbb{R}^{W}$ such that $(\mathbf{x}, \mathbf{y}) \in P$. Note that for every $\sigma \in \operatorname{ext}(\mathrm{id})$ it holds that $\left(\mathbf{x}, \mathbf{y}^{\sigma}\right) \in P$. Hence, we have $\left(\mathbf{x}, \mathbf{y}^{\prime}\right) \in P$ where $\mathbf{y}^{\prime}=\sum_{\sigma \in \operatorname{ext}(\mathrm{id})} \mathbf{y}^{\sigma} /|\operatorname{ext}(\mathrm{id})|$. Now for every $O \in \mathcal{O}$ let $p_{O}$ be the sum of the values taken by the variables from $O$ in the solution $(\mathbf{x}, \mathbf{y})$. In the solution $\left(\mathbf{x}, \mathbf{y}^{\prime}\right)$ every variable $y$ in $\mathcal{O}$ takes value $p_{O}\left|\operatorname{ext}(\mathrm{id})_{y}\right| /|\operatorname{ext}(\mathrm{id})|=$ $p_{O} /|\mathcal{O}|$, where $\operatorname{ext}(\mathrm{id})_{y}$ denotes the stabiliser of $y$ in ext(id). Hence, by assigning for every $O \in \mathcal{O}$ the value $p_{O}$ to the variable $y_{O}$ we obtain a point $(\mathbf{x}, \widehat{\mathbf{y}})$ in $\widehat{P}$. This implies that $A$ is contained in the subset of $\{0,1\}^{U}$ recognised by $\widehat{P}$. The other inclusion is clear.

We are now ready to state the main conclusion of this subsection.

Lemma 6. For every $G$-symmetric polytope $P$ of size s, there is a rigid $G$-symmetric polytope $Q$ which recognises the same set and the size of $Q$ is not more than $s \log (s)$.

Proof. First note that for any $G$-symmetric polytope $R$, if $R$ is not rigid, then $\widehat{R}$ has strictly fewer variables than $R$. Thus, starting at $P$, if we iterate the process, we must, in a finite number of steps reach a rigid polytope $Q$. For the size bound, note that the number of variables and constraints in $Q$ is at most the corresponding number in $P$. Moreover, each coefficient in $Q$ is the sum of distinct coefficients in $P$, of which there are at most $s$. Thus, if each coefficient in $P$ can be expressed with $b$ bits, each coefficient in $Q$ requires at most $b \log (s)$ bits and the bound follows.

\subsection{Bounded supports}

For a $\operatorname{Sym}_{n}$-set $Y$, a subset $S$ of $[n]$ is said to be a support of an element $y \in Y$ if for every $\pi \in \operatorname{Sym}_{n}$ that fixes $S$ pointwise, it holds that $\pi \cdot y=y$. Intuitively, this means that the action of the group $\operatorname{Sym}_{n}$ on $y$ depends only on the set $S$. We get even supports by replacing the symmetric group $\mathrm{Sym}_{n}$ by the alternating group $\mathrm{Alt}_{n}$. A subset $S$ of $[n]$ is said to be an 
even support of $y \in Y$ if for every $\pi \in \operatorname{Alt}_{n}$ that fixes $S$ pointwise, we have $\pi \cdot y=y$. Clearly, any support of $y$ is also an even support of $y$. Note also that every element of a $\mathrm{Sym}_{n}$-set is supported by the whole set $[n]$.

For a non-negative integer $k$, an (even) support $S$ is called $k$-bounded if $|S| \leq k$. A Sym $_{n^{-}}$ set $Y$ is called $k$-supported if each element of $Y$ has a $k$-bounded support. An $L$-symmetric polytope $P$ is called $k$-supported if the set of auxiliary variables and the set of constraints of $P$ are $k$-supported. We now show the following:

Lemma 7. There exists a positive integer $n_{0}$ such that for any positive integers $s$ and $n$ satisfying $s \geq n \geq n_{0}$, the following holds: If $P$ is a rigid L-symmetric LP lift of size $s$ for structures with $n$ elements, then $P$ is $k$-supported, where $k=O(\log (s) /(\log (n)-\log \log (s)))$. Moreover, if $s \leq 2^{n / 3}$, then the size of $P$ is at most $n^{k}$.

Proof. For the sake of simplicity we give the proof for the case when $L$ consists of a single binary symbol, that is for the case of graphs. The general case is completely analogous.

Consider a rigid graph-symmetric polytope lift $P \subseteq \mathbb{R}^{[n]^{2}} \times \mathbb{R}^{W}$ of size $s$ which recognises some property of graphs with $n$ vertices, and let $P$ be given by a sequence of linear constraints $\left(\gamma_{v}\right)_{v \in V}$ where each $\gamma_{v}$ is of the form $\mathbf{a}^{\mathrm{T}} \mathbf{x}+\mathbf{b}^{\mathrm{T}} \mathbf{y} \leq c$, with $\mathbf{x}=\left(x_{i j}\right)_{i, j \in[n]}$ and $\mathbf{y}=\left(y_{w}\right)_{w \in W}$. Since $P$ is rigid, it comes with an action of the group $\operatorname{Sym}_{n}$ on the set of auxiliary variables $\left\{y_{w}\right\}_{w \in W}$ such that for every $\pi \in \operatorname{Sym}_{n}$ we have $P^{\pi}=P$, and with an induced action of the group $\operatorname{Sym}_{n}$ on the set of constraints $\left\{\gamma_{v}\right\}_{v \in V}$.

If the size $s$ of the linear program $P$ is greater than $2^{n / 3}$, we can take $k=n$. Observe that in case $s>2^{n / 3}$ we have

$$
\frac{\log (s(n))}{\log (n)-\log \log (s(n))} \geq \frac{n / d_{0}}{\log (n)-\log (n)+\log (3)}=\frac{n}{3 \log (3)} .
$$

It follows that $k=O(\log (s) /(\log (n)-\log \log (s)))$. Since any element of a $\operatorname{Sym}_{n}$-set is supported by $[n]$, for this choice of $k$, every auxiliary variable and every constraint of $P$ has a $k$-bounded support.

In the case $s \leq 2^{n / 3}$ the argument is more involved. First we obtain bounded even supports. Take $t=\log (s) /(\log (n)-\log \log (s))$ and $k=\lceil t\rceil$. Observe that the denominator in the definition of $t$ is non-zero, since by assumption we have $s \leq 2^{n / 3}<2^{n}$. Also, we have $0<t \leq k \leq n / 3 \log (3)<n / 4<n / e$, to be used later in the proof. Let us start by noting that

$$
t \log \left(\frac{n}{t}\right)=\log (s) \frac{\log (n)-\log \log (s)+\log (\log (n)-\log \log (s))}{\log (n)-\log \log (s)}>\log (s) .
$$

The inequality follows from the fact that the big fraction in the middle is strictly bigger than 1 since $s \leq 2^{n / 3}$.

For any $S \subseteq[n]$, let $\operatorname{Alt}_{(S)}$ denote the group of all even permutations of $[n]$ that fix the set $S$ pointwise. We use the following fact which guarantees that subgroups of the symmetric group with small index contain as subgroups large alternating groups.

Lemma 8 (see Theorem 5.2B in [16]). If $n>8$ and $1 \leq k \leq n / 4$, and $G$ is a subgroup of $\operatorname{Sym}_{n}$ with $\left[\operatorname{Sym}_{n}: G\right]<\left(\begin{array}{l}n \\ k\end{array}\right)$, then there is a set $S \subseteq[n]$ with $|S|<k$ such that $\operatorname{Alt}_{(S)} \leq G$. 
For $w \in W$, let $\mathrm{St}_{w}$ denote the stabilizer of $y_{w}$ in $\mathrm{Sym}_{n}$, i.e., the subgroup of $\mathrm{Sym}_{n}$ defined by $\mathrm{St}_{w}=\left\{\pi \in \operatorname{Sym}_{n}: \pi \cdot y_{w}=y_{w}\right\}$. Since $\left[\mathrm{Sym}_{n}: \mathrm{St}_{w}\right]$ is the size of the orbit of $y_{w}$ under the action of $\mathrm{Sym}_{n}$ and the total number of auxiliary variables is bounded by the size of $P$, we have

$$
\left[\operatorname{Sym}_{n}: \mathrm{St}_{i}\right] \leq s<\left(\frac{n}{t}\right)^{t} \leq\left(\frac{n}{k}\right)^{k} \leq\left(\begin{array}{l}
n \\
k
\end{array}\right)
$$

with the second following from (23) , and the third from $0<t \leq k<n / 4<n / e$ and the fact that $f(x)=(n / x)^{x}$ is an increasing function of $x$ in the interval $(0, n / e)$. Lemma 8 implies that, if $n$ is large enough, there exist $S \subseteq[n]$ with $|S|<k$ and $\operatorname{Alt}_{(S)} \leq \mathrm{St}_{w}$. This is a $k$-bounded even support of $y_{w}$ in the way we defined. An entirely analogous argument yields a $k$-bounded even support for each constraint in $\left\{\gamma_{v}\right\}_{v \in V}$.

In order to obtain supports in place of even supports we need to introduce a way of looking at automorphism groups of symmetric polytopes as automorphism groups of graphs. In the following, whenever we talk about coloured vertices, by a colour we mean a graph gadget that forces the vertices of a colour to be mapped to vertices of the same colour by every possible automorphism.

Let $C$ be the set of all different numerical coefficients which appear in the linear constraints representing $P$. A graph representation of the polytope $P$ is a graph $\mathbb{P}$ with:

1. five disjoint sets of vertices: $[n],\left\{x_{i j}\right\}_{i, j \in[n]},\left\{y_{w}\right\}_{w \in W},\left\{\gamma_{v}\right\}_{v \in V}$ and $C$,

2. the vertices in $[n],\left\{x_{i j}\right\}_{i, j \in[n]},\left\{y_{w}\right\}_{w \in W}$ and $\left\{\gamma_{v}\right\}_{v \in V}$ coloured with four different colours depending on the set they belong to,

3. each vertex in $C$ coloured with a unique colour, which does not appear anywhere else in the graph,

4. for any $i, j \in[n]$, an edge from $i$ to $x_{i j}$ and from $j$ to $x_{i j}$,

5. for any $v \in V$ and any variable which appears in the constraint $\gamma_{v}$, a pair of edges: from the variable to its coefficient, and from this coefficient to $\gamma_{v}$,

6. for any $v \in V$, an edge from $\gamma_{v}$ to the vertex in $C$ corresponding to its constant term.

Observe that the automorphism group of the graph $\mathbb{P}$ is isomorphic to the automorphism group of $P$. Also, the number of vertices in $\mathbb{P}$ can be bounded by $O\left(s^{2}\right)$. This is because the number of vertices introduced in item (1) is at most $s$ and each gadget introduced in (2) and (3) can be chosen to have at most $2 s$ vertices and there are at most $s$ of them.

For any $S \subseteq[n]$, let $\operatorname{Sym}_{(S)}$ denote the group of all permutations of $[n]$ that fix the set $S$ pointwise. Take some $w \in W$ and let $S$ be a $k$-bounded even support of $y_{w}$. Since $\operatorname{Alt}_{(S)} \leq \operatorname{St}_{w}$, we have $\operatorname{Alt}_{(S)} \leq \operatorname{St}_{w} \cap \operatorname{Sym}_{(S)} \leq \operatorname{Sym}_{(S)}$. Hence, $\operatorname{St}_{w} \cap \operatorname{Sym}_{(S)}=\operatorname{Alt}_{(S)}$ or $\operatorname{St}_{w} \cap \operatorname{Sym}_{(S)}=\operatorname{Sym}_{(S)}$. We argue it is the latter case that holds using the following theorem which states that a graph whose automorphism group is the alternating group on an $n$-element set must be of size exponential in $n$.

Lemma 9 (Theorem $\mathrm{A}$ in [30]). If $n>22$, then the number of vertices of any graph whose full automorphism group is isomorphic to Alt $_{n}$ is at least $1 / 2\left(\begin{array}{c}n \\ \lfloor n / 2\rfloor\end{array}\right) \sim 2^{n} / \sqrt{2 \pi n}$. 
Assume that $\mathrm{St}_{w} \cap \operatorname{Sym}_{(S)}=\operatorname{Alt}_{(S)}$. We use Lemma 9 to arrive at a contradiction. Consider the graph representation $\mathbb{P}$ of the polytope $P$ modified in the following way: the vertices in $S \subseteq[n]$ and the vertex $y_{w}$ are coloured each with a different colour which did not appear in the graph before. Observe that the automorphism group of the graph $\mathbb{P}_{w}$ obtained this way is isomorphic to $\mathrm{St}_{w} \cap \mathrm{Sym}_{(S)}$, and therefore isomorphic to $\operatorname{Alt}_{(S)}$, which in turn is isomorphic to the alternating group on the set $[n-|S|]$. And, once again, the number of vertices of $\mathbb{P}_{w}$ is $O\left(s^{2}\right)$. Thus, if $n$ is large enough, we have

$$
s^{2}<2^{2 n / 3}<\frac{1}{2}\left(\begin{array}{c}
n \\
\lfloor n / 2\rfloor
\end{array}\right) .
$$

Hence, by Lemma 9, we obtain the desired contradiction.

From $\mathrm{St}_{w} \cap \operatorname{Sym}_{(S)}=\operatorname{Sym}_{(S)}$ it follows that $\operatorname{Sym}_{(S)} \leq \mathrm{St}_{w}$, which means that $S$ is a $k$-bounded support of $y_{w}$ in the way we defined. An analogous argument yields a $k$-bounded support for each linear constraint in $\left\{\gamma_{v}\right\}_{v \in V}$. Note also that that $s<\left(\begin{array}{l}n \\ k\end{array}\right) \leq n^{k}$. In particular, $P$ has at most $n^{k}$ auxiliary variables, at most $n^{k}$ constraints, and all its coefficients and constant terms can be written down using at most $n^{k}$ bits.

We now show that it is possible to (non-uniquely) represent the auxiliary variables and constraints of $k$-supported polytopes by tuples of integers from $[n]$ of length $k$ in a way that is consistent with the group action. In order for the representation to be uniform across all $n$, we extend the definition of the set $[n]^{(k)}$ to the case when $k>n$. For positive integers $n$ and $k$ such that $k>n$, the set $[n]^{(k)}$ consists of $k$-tuples of elements of $[n]$ with the first $n$ components pairwise distinct and the last $k-n$ components equal to the $n$-th component. In particular, if $k>n$, then every tuple in $[n]^{(k)}$ contains all the elements of $[n]$.

For any positive integer $n$ and any non-negative integer $k$, we consider the set $[n]^{(k)}$ as a $\mathrm{Sym}_{n}$-set with the natural action of the group $\mathrm{Sym}_{n}$. Note that this $\mathrm{Sym}_{n}$-set has one orbit. Indeed, if we take any $k$-tuples $\mathbf{s}_{1}$ and $\mathbf{s}_{2}$ from $[n]^{(k)}$, then there exists a permutation $\pi$ such that $\pi \cdot \mathbf{s}_{1}=\mathbf{s}_{2}$. This is because the equality types of any two elements of $[n]^{(k)}$ are the same.

Lemma 10. Let $Y$ be a single-orbit $k$-supported $\mathrm{Sym}_{n}$-set. There is a surjective homomorphism from $[n]^{(k)}$ to $Y$.

Proof. Take any $y \in Y$ and let $S$ be a $k$-bounded support of $y$. Since a superset of a support is a support itself, without loss of generality we can assume that $|S|=\min \{k, n\}$. Now, pick a tuple $\mathbf{s} \in S^{(k)}$. If $k \leq n$, then $|S|=k$ and every element of $S$ appears exactly once in the tuple $\mathbf{s}$, otherwise the tuple $\mathbf{s}$ contains all the elements of $[n]$.

We define a homomorphism $f$ from the $\operatorname{Sym}_{n}$-set $[n]^{(k)}$ to the $\operatorname{Sym}_{n}$-set $Y$ which for any $\pi \in \operatorname{Sym}_{n}$ maps $\pi \cdot \mathbf{s}$ to $\pi \cdot y$. The only thing that needs to be verified is whether the function $f$ is well defined. To this end, suppose that for some permutations $\pi_{1}, \pi_{2} \in \mathrm{Sym}_{n}$ it holds that $\pi_{1} \cdot \mathbf{s}=\pi_{2} \cdot \mathbf{s}$. Then $\pi_{2}^{-1} \pi_{1} \cdot \mathbf{s}=\mathbf{s}$, that is, the permutation $\pi_{2}^{-1} \pi_{1}$ fixes the support $S$ of $y$ pointwise. Therefore, $\pi_{2}^{-1} \pi_{1} \cdot y=y$ which implies that $\pi_{1} \cdot y=\pi_{2} \cdot y$. Since $Y$ has one orbit, the homomorphism $f$ is surjective. 
Once a surjective homomorphism $f$ from a $\operatorname{Sym}_{n}$-set $[n]^{(k)}$ to a $\operatorname{Sym}_{n}$-set $Y$ is fixed, the family $\left\{f^{-1}(y)\right\}_{y \in Y}$ forms a partition of $[n]^{(k)}$. Hence, for any $y \in Y$, each tuple $\left(i_{1}, \ldots, i_{k}\right)$ from $f^{-1}(y)$ uniquely identifies $y$, and is called an identifier of $y$ (with respect to the homomorphism $f$ ). In most cases each element of $Y$ has several identifiers.

Let us illustrate Lemma 10 by an example. For $n \geq 2$, consider the set $Y$ of twoelement subsets of $[n]$ with the natural action of the group $\operatorname{Sym}_{n}$, i.e., for any $\pi \in \operatorname{Sym}_{n}$ and any distinct $i, j \in[n]$, we have $\pi \cdot\{i, j\}=\{\pi(i), \pi(j)\}$. This single-orbit $\operatorname{Sym}_{n}$-set is $k$-supported, for any $k \geq 2$. Let us take $k=2$. The homomorphism from Lemma 10 is then unique and given by $(i, j) \mapsto\{i, j\}$. Note that the inverse image of any $\{i, j\} \in Y$ has two elements, and hence, each element $\{i, j\}$ of $Y$ has two identifiers: $(i, j)$ and $(j, i)$. For $k=3$, applying Lemma 10 yields several different homomorphisms. If $n \geq 3$, one of them is given by $(i, j, *) \mapsto\{i, j\}$, where by $*$ we mean any element of $[n]$ distinct from both $i$ and $j$. In this case, the inverse image of any $\{i, j\} \in Y$ has $2 n-4$ elements. For $n=2$ and $k=3$, the homomorphism is again unique and given by $(i, j, j) \mapsto\{i, j\}$ yielding two identifiers, for every element of $Y$.

To give one more example, consider a single-orbit set which consists of a single element $y$ with the trivial action of the symmetric group $\operatorname{Sym}_{n}$. This set is $k$-supported, for any non-negative integer $k$. For any $k \geq 0$, the homomorphism from Lemma 10 mapa each tuple from $[n]^{(k)}$ to $y$. In particular, for $k=0$, we have $\epsilon \mapsto y$, where $\epsilon$ is the only element of $[n]^{(0)}$, that is, the empty tuple.

To represent elements of a $k$-supported $\mathrm{Sym}_{n}$-sets with potentially more than one orbit, we need to introduce several copies of the set $[n]^{(k)}$, one for each orbit.

Corollary 1. Let $Y$ be a k-supported $\mathrm{Sym}_{n}$-set. There is a surjective homomorphism from $Q \times[n]^{k}$ to $Y$, where the size of $Q$ is equal to the number of orbits of $Y$.

It is clear how to extend the definition of an identifier to the general case discussed in the corollary above. Note that if a tuple $\left(q, i_{1}, \ldots, i_{k}\right)$ is an identifier of $y \in Y$, then the tuple $\left(q, \pi\left(i_{1}\right), \ldots, \pi\left(i_{k}\right)\right)$ is an identifier of $\pi \cdot y$.

\subsection{Manageable polytopes}

For a non-negative integer $k$, a polytope $P$ over $\mathbb{R}^{L(n)} \times \mathbb{R}^{W}$ is called $k$-manageable if:

1. there are two sets $Q$ and $T$ with a trivial action of the group $\operatorname{Sym}_{n}$,

2. the set of constraints of $P$ is indexed by $V=Q \times[n]^{k}$,

3. the set of auxiliary variables of $P$ is indexed by $W=T \times[n]^{k}$,

4. $P$ is $L$-symmetric with respect to the natural action of $\mathrm{Sym}_{n}$ on $W$, and the induced action of $\mathrm{Sym}_{n}$ on the set of constraints is exactly the natural action of $\mathrm{Sym}_{n}$ on $V$.

An example of a manageable polytope is given at the very beginning of this section. There the set of auxiliary variables has only one orbit $\left\{y_{12}, y_{21}\right\}$ so introducing $T$ is not necessary.

The key property of $k$-manageable polytopes, which allows us to use them in the translation from families of linear programs to logic, is the following. 
Lemma 11. If $P$ is a $k$-manageable polytope with constraints indexed by $V=Q \times[n]^{(k)}$ and auxiliary variables indexed by $W=T \times[n]^{(k)}$, then for any $R \in L, q \in Q, t \in T$, $\mathbf{i}, \mathbf{i}^{\prime}, \mathbf{j}, \mathbf{j}^{\prime} \in[n]^{(k)}, \mathbf{k}, \mathbf{k}^{\prime} \in[n]^{\operatorname{ar}(R)}$ :

1. the constant terms of the linear constraints $\gamma_{(q, \mathbf{i})}$ and $\gamma_{\left(q, \mathbf{i}^{\prime}\right)}$ are the same,

2. if the equality types of the tuples $(\mathbf{j}, \mathbf{i})$ and $\left(\mathbf{j}^{\prime}, \mathbf{i}^{\prime}\right)$ are the same, then the coefficient of the variable $y_{(t, \mathbf{j})}$ in the linear constraint $\gamma_{(q, \mathbf{i})}$ is the same as the coefficient of the variable $y_{\left(t, \mathbf{j}^{\prime}\right)}$ in the linear constraint $\gamma_{\left(q, \mathbf{i}^{\prime}\right)}$,

3. if the equality types of the tuples $(\mathbf{k}, \mathbf{i})$ and $\left(\mathbf{k}^{\prime}, \mathbf{i}^{\prime}\right)$ are the same, then the coefficient of the variable $x_{(R, \mathbf{k})}$ in the linear constraint $\gamma_{(q, \mathbf{i})}$ is the same as the coefficient of the variable $x_{\left(R, \mathbf{k}^{\prime}\right)}$ in the linear constraint $\gamma_{\left(q, \mathbf{i}^{\prime}\right)}$.

Proof. 1. Recall that every tuple in the set $[n]^{(k)}$ has the same equality type. Therefore, there exists a permutation $\pi \in \operatorname{Sym}_{n}$ which maps the tuple $\mathbf{i}$ to $\mathbf{i}^{\prime}$. Since $\gamma_{(q, \mathbf{i})}^{\pi}=\gamma_{\left(q, \mathbf{i}^{\prime}\right)}$, the constant terms in the linear constraints $\gamma_{(q, \mathbf{i})}$ and $\gamma_{\left(q, \mathbf{i}^{\prime}\right)}$ are the same.

2. If the equality types of the tuples $(\mathbf{j}, \mathbf{i})$ and $\left(\mathbf{j}^{\prime}, \mathbf{i}^{\prime}\right)$ are the same, then there exists a permutation $\pi \in \operatorname{Sym}_{n}$, which maps $\mathbf{j}$ to $\mathbf{j}^{\prime}$, and $\mathbf{i}$ to $\mathbf{i}^{\prime}$. Let $a$ be the coefficient of the variable $y_{(t, \mathbf{j})}$ in the linear constraint $\gamma_{(q, \mathbf{i})}$. By applying the permutation $\pi$ to the constraint $\gamma_{(q, \mathbf{i})}$, we get the constraint $\gamma_{\left(q, \mathbf{i}^{\prime}\right)}$. Moreover, since $\pi \cdot y_{(t, \mathbf{j})}=y_{\left(t, \mathbf{j}^{\prime}\right)}$, the coefficient of the variable $y_{\left(t, \mathbf{j}^{\prime}\right)}$ in $\gamma_{\left(q, \mathbf{i}^{\prime}\right)}$ is $a$. The proof of 3 . is analogous.

Now, suppose that a $k$-supported rigid $L$-symmetric LP lift $P \subseteq \mathbb{R}^{L(n)} \times \mathbb{R}^{W}$ recognizes some property of $L$-structures, that is, a subset $A$ of $\{0,1\}^{L(n)}$. We argue that there exists a $k$-manageable polytope lift $\bar{P}$ recognising $A$. Since the polytope $P$ is $k$-supported, by applying Lemma [10 we obtain two sets of identifiers: $\bar{V}=Q \times[n]^{k}$ for the constraints, and $\bar{W}=T \times[n]^{k}$ for the auxiliary variables. Let us introduce a new variable of the form $y_{(t, \mathbf{j})}$, for any identifier $(t, \mathbf{j}) \in \bar{W}$. We obtain a manageable polytope $\bar{P}$ from the polytope $P$ by first, replacing, for each $w \in W$, the auxiliary variable $y_{w}$ in $P$ by the sum of variables $y_{(t, \mathbf{j})}$ over the set of all identifiers $(t, \mathbf{j})$ of $y_{w}$; and secondly, replacing, for every $v \in V$, the constraint $\gamma_{v}$, by several copies of this constraint, one for every identifier $(q, \mathbf{i})$ of $\gamma_{v}$. The obtained polytope lift $\bar{P}$ is clearly $k$-manageable and it is easy to see that it recognizes the same property of $L$-structures.

To summarize, the constructions in Subsections 4.1, 4.2 and 4.3 imply that if a property of $L$-structures is recognised by a family of $L$-symmetric LP lifts of size $s(n)$, then the same property is recognised by a family of $k(n)$-manageable LP lifts, where $k(n)=$ $O(\log (s(n)) /(\log (n)-\log \log (s(n))))$.

\subsection{From manageable polytopes to counting logic}

We now put everything together in the proof of Lemma 5. For the sake of simplicity we give the proof for the case when $L$ consists of a single binary symbol, that is for the case of graphs. The general case is completely analogous. 
Let $P \subseteq \mathbb{R}^{[n]^{2}} \times \mathbb{R}^{W}$ be a graph-symmetric LP lift of size $s$ recognising some property of graphs with $n$ vertices, that is, a subset $A$ of $\{0,1\}^{[n]^{2}}$. We show that the same property of graphs is definable by a $\mathrm{C}^{k}$ formula, where $k=O(\log (s) /(\log (n)-\log \log (s)))$.

Let $\widehat{P}$ be a rigid graph-symmetric LP lift recognising $A$, as constructed in Subsection 4.1 , Recall that its size $s^{\prime}$ is at most $s \log (s)$ where $s$ is the size of $P$. In particular, $s^{\prime} \leq s^{2}$.

If $s>2^{n / 6}$, by a calculation analogous to (22) at the beginning of Subsection 4.2, we have $n=O(\log (s) /(\log (n)-\log \log (s)))$. Since every class of graphs with $n$ vertices is definable in $\mathrm{C}^{n}$, we complete the proof of the lemma in this case by taking $k=n$.

If $s \leq 2^{n / 6}$, then $s^{\prime} \leq s^{2} \leq 2^{n / 3}$. Hence, by Lemma 7, for some $k=O\left(\log \left(s^{\prime}\right) /(\log (n)-\right.$ $\left.\left.\log \log \left(s^{\prime}\right)\right)\right), \widehat{P}$ is $k$-supported, has at most $n^{k}$ auxiliary variables, at most $n^{k}$ constraints, and all its coefficients and constant terms can be encoded using at most $n^{k}$ bits. Moreover, any such $k$ clearly satisfies $k=O(\log (s) /(\log (n)-\log \log (s)))$.

Let $\bar{P}$ be a $k$-manageable polytope lift recognising $A$ (as described in Subsection 4.3) with the set of constraints indexed by $Q \times[n]^{k}$, and the set of auxiliary variables indexed by $T \times[n]^{k}$. Note that it follows from the construction of $\bar{P}$ that the number of elements in the sets $T$ and $Q$ is bounded, respectively, by the number of auxiliary variables and the number of constraints in $\widehat{P}$. Hence, $|Q|,|T| \leq n^{k}$.

Suppose now that we are given a graph $G$ with the set of vertices $V$ of size $n$ and the set of edges $E$. Intuitively, if we could fix a bijection between $[n]$ and $V$, we could then compute from $\bar{P}$ and $G$ a linear program $\bar{P}_{G}$ with the set of constraints $I$ and the set of variables $J$ as follows:

$$
\begin{aligned}
& I=\left\{\gamma_{(q, \mathbf{v})}: q \in Q, \mathbf{v} \in V^{k}\right\}, \\
& J=\left\{x_{v w}: v, w \in V\right\} \cup\left\{y_{(t, \mathbf{v})}: t \in T, \mathbf{v} \in V^{k}\right\} .
\end{aligned}
$$

In order to decide whether $G$ has the property of interest we would then check if the partial valuation: $x_{v w}=1$ if $(v, w) \in E$, and $x_{v w}=0$ otherwise, can be extended to a full solution. This in turn can be easily done in logic using the following straightforward consequence of the results in [4].

Lemma 12. There exists an FPC formula $\phi$ which given a matrix $\mathbf{A} \in \mathbb{Q}^{I \times J}$ and a pair of vectors $\mathbf{b} \in \mathbb{Q}^{I}$, and $\mathbf{a} \in \mathbb{Q}^{J^{\prime}}$, where $J^{\prime} \subseteq J$, decides if $\mathbf{a}$ can be extended to a solution of the linear program $\mathbf{A} \mathbf{x} \leq \mathbf{b}$.

Our goal is to use Lemma 11 to show that the linear program $\bar{P}_{G}$ can be computed without fixing a bijection between $[n]$ and $V$. We define an FOC-interpretation $\Psi$ which takes as input a graph $G$ with $n$ vertices and outputs, essentially, a relational encoding of the linear program $\bar{P}_{G}$ together with the partial valuation discussed above. More precisely, $\Psi$ outputs a matrix $\mathbf{A} \in \mathbb{Q}^{I \times J}$ and a pair of vectors $\mathbf{b} \in \mathbb{Q}^{I}$, and $\mathbf{a} \in \mathbb{Q}^{J^{\prime}}$, where $J^{\prime} \subseteq J$, such that $\mathbf{a}$ can be extended to a solution of $\mathbf{A x} \leq \mathbf{b}$ if and only if $G$ has the property of interest. To encode the fact that $J^{\prime} \subseteq J$ we introduce an extra binary relation symbol $F$ of type $\bar{J}^{\prime} \times \bar{J}$ for an injective function from the index set $J^{\prime}$ to the index set $J$. Hence, $\Psi$ outputs a structure over the vocabulary $L_{\mathrm{vec}, 2} \cup L_{\mathrm{vec}, 1} \dot{\cup} L_{\mathrm{vec}, 1} \cup \dot{\cup}\{F\}$ modified, for simplicity, in such a way that there is a single sort symbol $\bar{B}$ for a domain of bit positions. 
Given a graph $G$ with $n$ vertices the FOC-interpretation $\Psi$ has access to the domain $V$ of the graph, and the naturally ordered number domain $\{0, \ldots, n\}$. To represent the bit encodings of the numerical coefficients we use tuples from $[n]^{k} \subseteq\{0, \ldots, n\}^{k}$. Let $o:[n]^{k} \rightarrow$ $\left\{0,1, \ldots, n^{k}-1\right\}$ be the order-preserving bijection from the set $[n]^{k}$ ordered lexicographically to the set $\left\{0,1, \ldots, n^{k}-1\right\}$ with the natural order. For any $\mathbf{s} \in[n]^{k}$, by $[\mathbf{s}]$ we denote the natural number $o(\mathbf{s})$. Tuples from $[n]^{k} \subseteq\{0, \ldots, n\}^{k}$ are also used to represent elements of $Q$ and $T$. In order to do so let us fix an injective function $f$ from $Q$ to $[n]^{k}$, and an injective function $g$ from $T$ to $[n]^{k}$. The linear program $\mathbf{A x} \leq \mathbf{b}$ in the output of $\Psi$ has the set of constraints indexed by $[n]^{k} \times V^{k}$ and the set of variables indexed by $V^{2} \cup[n]^{k} \times V^{k}$. Once restricted to the constraints indexed by $f(Q) \times V^{k}$ and the variables indexed by $V^{2} \cup g(T) \times V^{k}$ it is exactly the linear program $\bar{P}_{G}$. All the other coefficients and constant terms in $\mathbf{A x} \leq \mathbf{b}$ are set to $0=(-1)^{0} 0 / 1$.

Consider tuples of the form $\left(\mathbf{z}_{1}, \mathbf{z}_{2}, \mathbf{z}_{3}, \rho\right)$, where $\mathbf{z}_{1}, \mathbf{z}_{2}, \mathbf{z}_{3} \in[n]^{k}$, and $\rho$ is a quantifier-free formula defining an equality type of $2 k$-tuples. By $T_{d}^{y}$ let us denote the set of all tuples of this form which satisfy one of the following conditions:

- $\mathbf{z}_{1} \notin f(Q)$ or $\mathbf{z}_{2} \notin g(T)$, and $\left[\mathbf{z}_{3}\right]=0$,

- $\mathbf{z}_{1} \in f(Q)$ and $\mathbf{z}_{2} \in g(T)$, and if $f^{-1}\left(\mathbf{z}_{1}\right)=q, g^{-1}\left(\mathbf{z}_{2}\right)=t$, then for every $\mathbf{s}_{1}, \mathbf{s}_{2} \in[n]^{k}$ such that the equality type of $\left(\mathbf{s}_{1}, \mathbf{s}_{2}\right)$ is $\rho$, the position $\left[\mathbf{z}_{3}\right]$ in the binary encoding of the denominator of the coefficient of the variable indexed by $\left(t, \mathbf{s}_{2}\right)$ in the constraint indexed by $\left(q, \mathbf{s}_{1}\right)$ in $\bar{P}$ carries the 1-bit.

It follows from Lemma 11 that the set $T_{d}^{y}$ carries all information about the denominators of the coefficients of the auxiliary variables in $\bar{P}$.

Similarly, we define sets $T_{s}^{y}, T_{n}^{y}, T_{s}^{x}, T_{n}^{x}, T_{d}^{x}$, and $C_{s}, C_{n}, C_{d}$ to carry all the information about the signs and the bits of the numerators and the denominators of: the coefficients of the auxiliary variables, the coefficients of the variables in $\left\{x_{i j}\right\}_{1 \leq i, j \leq n}$, and the constant terms, respectively.

Given a graph $G$ with the set of vertices $V$ of size $n$ and the set of edges $E$ the interpretation $\Psi$ does the following:

1. defines the domain of $\bar{I}$ as $[n]^{k} \times V^{k}$, the domain of $\bar{J}$ as $V^{2} \cup[n]^{k} \times V^{k}$, the domain of $\bar{J}^{\prime}$ as $V^{2}$, and the domain of $\bar{B}$ as $[n]^{k}$, where $V$ is the domain of $G$, and $[n] \subseteq\{0, \ldots, n\}$ is a subset of the number domain,

2. defines the relation $\leq$ for the linear order on $\bar{B}$ as the lexicographic order with respect to the natural order of the number domain,

3. defines the relation $F$ of type $\bar{J}^{\prime} \times \bar{J}$ as the equality relation on $V^{2}$,

4. defines the ternary relation $P_{d}^{\mathbf{A}}$ of type $\bar{I} \times \bar{J} \times \bar{B}$ for encoding the denominators of the entries of the matrix $\mathbf{A}$ as a union of two relations. The first is defined as a subset of $\left([n]^{k} \times V^{k}\right) \times\left([n]^{k} \times V^{k}\right) \times[n]^{k}$ consisting of tuples $\left(\mathbf{s}_{1}, \mathbf{v}_{1}, \mathbf{s}_{2}, \mathbf{v}_{2}, \mathbf{s}_{3}\right)$ for which there exists $\left(\mathbf{z}_{1}, \mathbf{z}_{2}, \mathbf{z}_{3}, \rho\right)$ in $T_{d}^{y}$ such that the tuple $\left(\mathbf{v}_{1}, \mathbf{v}_{2}\right)$ satisfies $\rho$, and for every $i \in[3]$ it holds $\mathbf{s}_{i}=\mathbf{z}_{i}$. The second is defined as a subset of $\left([n]^{k} \times V^{k}\right) \times V^{2} \times[n]^{k}$ consisting 
of tuples $\left(\mathbf{s}_{1}, \mathbf{v}_{1}, v, w, \mathbf{s}_{2}\right)$ for which there exists $\left(\mathbf{z}_{1}, \mathbf{z}_{2}, \rho\right)$ in $T_{d}^{x}$ such that the tuple $\left(\mathbf{v}_{1}, v, w\right)$ satisfies $\rho$ and $\mathbf{s}_{1}=\mathbf{z}_{1}$, and $\mathbf{s}_{2}=\mathbf{z}_{2}$,

5. defines the relations $P_{s}^{\mathbf{A}}, P_{n}^{\mathbf{A}}, P_{s}^{\mathbf{b}}, P_{n}^{\mathbf{b}}, P_{d}^{\mathbf{b}}$ in a similar way as $P_{n}^{\mathbf{A}}$,

6 . defines the binary relations $P_{s}^{\mathbf{a}}, P_{n}^{\mathbf{a}}, P_{d}^{\mathbf{a}}$ of type $\bar{J}^{\prime} \times \bar{B}$ for encoding the entries of the vector a in the following way: the entries $((v, w), \mathbf{s})$ of $P_{s}^{\mathbf{a}}, P_{n}^{\mathbf{a}}, P_{d}^{\mathbf{a}}$ are defined to encode $1=(-1)^{0} 1 / 1$ or $0=(-1)^{0} 0 / 1$ depending on whether $(v, w) \in E$ or not.

Note that by existential quantification over the sets $T_{d}^{y}$ and $T_{d}^{x}$ we really mean a disjunction. And by $\mathbf{s}_{i}=\mathbf{z}_{i}$ we mean the 2-variable FO-formula of size $O(k n)$ which, for every $j \in[k]$, says that the $j$-th component $s_{i, j}$ of $\mathbf{s}_{i}$ is the $z_{i, j}$-th component of $[n]$, using the order on the number domain. Observe also that $\Psi$, as described, is not rigorously an FOC-interpretation, but it is not difficult to see that it can be easily turned into such.

The interpretation $\Psi$ has $O(k)$ variables. Its size is polynomial in $n^{k}$, in $k$, and in the number of equality types of $2 k$ tuples, that is, polynomial in $n^{k}, k$, and $(2 k)^{2 k}$. Since in our case $k=O(n)$, the size of $\Psi$ is simply $n^{O(k)}$.

Now by composing $\Psi$ with the FPC formula $\phi$ from Lemma 12 we obtain an FPC formula $\psi$ which given a graph $G$ with $n$ vertices decides if $G$ has the property of interest. The formula $\psi$ has $l=O(k)$ variables and size $n^{O(k)}$. We translate it into a formula $\theta$ of $\mathrm{C}^{2 l}$ such that $\psi$ is equivalent to $\theta$ on all structures of size at most $n$ and $\theta$ is of size polynomial in the size of $\psi$, in $l$, and in $n^{l}$ (cf. Subsection 2.2). Hence, in terms of $k$ and $n$, the formula $\theta$ has $O(k)$ variables and size $n^{O(k)}$.

We have therefore shown that a property of graphs with $n$ vertices recognized by a graphsymmetric polytope lift of size $s$ is defined by a $C^{k}$ formula, where $k=O(\log (s) /(\log (n)-$ $\log \log (s)))$. Moreover, if $s$ is at most weakly exponential, then for some positive real $\epsilon$ we have $k=O(\log (s) /(\log (n)-\log \log (s)))=O(\log (s) /(\epsilon \log (n)))=O(\log (s) / \log (n))$. Hence, in this case the size of $\theta$ is $n^{O(k)}=s^{O(1)}$. This finishes the proof of Lemma 5 and this section.

\section{$5 \quad$ Results and Applications}

In this section we develop the main consequences of our results. We start by establishing the main theorem of the paper, which characterizes the expressive power of symmetric linear programs. We continue with the applications to upper and lower bounds. And end with the observation that for random graphs over appropriate distributions symmetric LP lifts are as powerful as general Boolean circuits.

\subsection{Equivalence of Models}

If $\mathscr{C}$ is a class of finite $L$-structures of some single-sorted vocabulary $L$, and $n$ is a positive integer, we write $\mathscr{C}_{n}$ for the set of all structures in $\mathscr{C}$ of cardinality $n$. We write se्C $(n)$ for the size of a smallest $L$-symmetric Boolean circuit that recognizes $\mathscr{C}_{n}$, and $\operatorname{lp}_{\mathscr{C}}(n)$ for the size of a smallest $L$-symmetric LP lift that recognizes $\mathscr{C}_{n}$. Similarly, we write $\mathrm{w}_{\mathscr{C}}(n)$ for the counting-width of $\mathscr{C}_{n}$, i.e., the smallest number of variables $k$ of a $\mathrm{C}^{k}$-formula that defines $\mathscr{C}_{n}$ 
on $L$-structures of cardinality $n$, and $\operatorname{sw}_{\mathscr{C}}(n)$ for the counting size-width of $\mathscr{C}_{n}$, i.e., the smallest $k$ such that there is a $\mathrm{C}^{k}$-formula of size at most $n^{k}$ that defines $\mathscr{C}_{n}$ on $L$-structures of cardinality $n$.

Theorem 1. Let $\mathscr{C}$ be a class of finite L-structures of some vocabulary L. If $\operatorname{lp}_{\mathscr{C}}(n)$ is at most weakly exponential, then

$$
\begin{aligned}
& \text { 1. } \mathrm{s}_{\mathscr{C}}(n)^{\Omega(1)} \leq \operatorname{lp}_{\mathscr{C}}(n) \leq \mathrm{s}_{\mathscr{C}}(n)^{O(1)} \\
& \text { 2. } \Omega\left(\operatorname{sw}_{\mathscr{C}}(n)\right) \leq \log \left(\operatorname{lp}_{\mathscr{C}}(n)\right) / \log (n) \leq O\left(\operatorname{sw}_{\mathscr{C}}(n)\right) .
\end{aligned}
$$

Proof. The upper bound in 1 is a direct consequence of Lemma 1, and this holds without any assumption on the growth rate of $\operatorname{lp}_{\mathscr{C}}(n)$. The lower bound in 2 follows from Lemma 5. Write $s=\operatorname{lp}_{\mathscr{C}}(n)$ and choose $k=c \log (s) /(\log (n)-\log \log (s))$ for a large $c$ to be specified later. Since $\operatorname{lp}_{\mathscr{C}}(n)$ is at most weakly exponential we have $s \leq 2^{n^{1-\epsilon}}$ for some $\epsilon>0$ and large enough $n$. Hence $k=O(\log (s) / \log (n))$ with the hidden constant in the big-oh notation dependent on $\epsilon$ as in $1 / \epsilon$. Now, for the appropriate constant in the big-oh in $k=O(\log (s) / \log (n))$, Lemma 5 says that there is a $\mathrm{C}^{k}$-formula that defines $\mathscr{C}$ and has size polynomial in $s$, since again $\operatorname{lp}_{\mathscr{C}}(n)$ is at most weakly exponential. If the constant in the big-oh in $k=O(\log (s) / \log (n))$ is chosen big enough, we get that the size polynomial in $s$ is bounded even by $n^{k}$, so $\operatorname{sw}_{\mathscr{C}}(n)=O(\log (s) / \log (n))$ as was to be proved. In turn, these two imply the lower bound in 1 and the upper bound in 2 through the well-known relationship $\mathrm{s}_{\mathscr{C}}(n) \leq n^{O\left(\mathrm{sw}_{\mathscr{C}}(n)\right)}($ see $[33])$.

\subsection{Upper and Lower Bounds}

By Theorem 1, any class of graphs of unbounded counting width cannot be recognized by polynomial-size symmetric LP lifts. More strongly, in combination with the strongest known lower bounds on counting width, Theorem 1 gives weakly exponential lower bounds of the type $2^{\Omega\left(n^{1-\epsilon}\right)}$. We show that the strongest forms of Lemmas 1 and 5 give even larger lower bounds.

Lower bounds on symmetric lifts and circuits In the sequel, let 3-XOR refer to the constraint satisfaction problem of deciding whether a system of 3-variable parity constraints on $\{0,1\}$-valued variables is satisfiable, and let 3-SAT refer to the satisfiability problem for 3$\mathrm{CNF}$ formulas. In both cases, an instance is presented as a finite structure that encodes the incidence structure of the constraints: the domain is the disjoint union of the set of variables and the set of constraints, and the relations carry one monadic relation for each type of constraint that indicates which constraints are of that type, and three binary relations that indicate the three variables that participate in each constraint. Note that the instances for these problems are not plain graphs but graphs with coloured vertices and edges.

Theorem 2. Every graph-symmetric LP lift or Boolean threshold circuit that recognizes the class of Hamiltonian graphs with $n$ vertices, or the class of 3-colourable graphs with $n$ vertices, or the class of satisfiable 3-SAT instances with $n$ variables, or the class of satisfiable 3-XOR 
instances with $n$ variables, has size $2^{\Omega(n)}$. Moreover, for 3-colouring, 3-SAT, and 3-XOR, the lower bound holds even on the class of instances with $O(n)$ edges, $O(n)$ clauses, and $O(n)$ constraints, respectively.

Before we enter the proof let us note that these $2^{\Omega(n)}$ lower bounds for 3-colouring, 3-XOR and 3-SAT are optimal up to the multiplicative constant in the exponent. We discuss this later in this section. Now we turn to the proof of Theorem 2. First we handle 3-colourability, then Hamiltonicity. As intermediate steps towards both we do 3-XOR and 3-SAT.

By Lemma 5, for obtaining the lower bound for LP lifts it suffices to show that any $\mathrm{C}^{k}$ sentence that defines the class of $n$-vertex 3-colourable graphs has $k=\Omega(n)$ : indeed, whenever $s \leq 2^{n / d}$, we have

$$
\log (s) /(\log (n)-\log \log (s)) \leq n /(d \log (d))
$$

By Lemma 1, the claim then follows for Boolean threshold circuits. A result from the literature that is quite close to the $k=\Omega(n)$ that we need can be found in Section 4.2 in [13], but the analysis in there gives $k=\Omega(\sqrt{n})$, and not $k=\Omega(n)$. While it should be possible to modify the construction in [13] to get what we need, we refer to a more recent construction that achieves what we want for the problems 3-XOR and 3-SAT, and then proceed by reduction. These intermediate steps will also be useful when we discuss Hamiltonicity.

Theorem 3 (see Theorem 3.7 and 3.8 in [5] and Lemmas 22 and 23 in [15]). There exist $c, d>0$ such that, for every $k$ and every sufficiently large $n$, every $\mathrm{C}^{k}$-sentence that separates the class of satisfiable 3-XOR (resp. 3-SAT) instances with $n$ variables and cn constraints from the class of unsatisfiable ones has $k \geq d n$.

Neither [5] nor [15] state the linear bound $c n$ on the number of constraints, but it easily follow from both proofs. Concretely, it follows from Lemma 3.3 in [5], in which the bound $i s$ stated. Now we proceed by reduction in order to get the same result for 3-colouring:

Lemma 13. There exist $c, d>0$ such that, for every $k$ and every sufficiently large $n$, every $\mathrm{C}^{k}$-sentence that separates the class of 3-colourable graphs with $n$ vertices and cn edges from the class of non-3-colourable ones has $k \geq d n$.

Proof. In the textbook reduction from 3-SAT to the problem of deciding whether a graph is 3colourable (see, e.g., [34]), the output graph has one gadget with two vertices for each variable in the input formula, one gadget with six vertices for each constraint of the input formula (the reduction in [34] uses only three vertices for each constraint because the reduction starts at NAE-SAT; starting at 3-SAT we need six vertices), and one special vertex. The edges are local to each gadget, plus two edges from each variable gadget to the special vertex, and a constant number of edges for each variable occurrence in the input formula between the constraint gadget where the variable appears, and the corresponding variable gadget. There are no other vertices or edges in the graph. It is clear from the construction that if the input formula has $n$ vertices and $m$ constraints, then this graph has $O(n)+O(m)$ vertices and 
$O(n)+O(m)+O(m)$ edges, since each of the $m$ constraints contributes three occurrences. And it is not difficult to see that any $\mathrm{C}^{k}$ formula that separates the 3-colourable graphs that are output by the reduction from the non-3-colourable ones can be converted into a $\mathrm{C}^{O(k)}$ formula that separate the satisfiable 3-SAT instances that are input to the reduction from the unsatisfiable ones. Another way to see this is by noting that the reduction is definable by a uniform quantifer-free interpretation (without the need for any ordering, or parameters, on the input structure), from which the claim on $\mathrm{C}^{O(k)}$-definability follows from the closure of the logic under quantifier-free interpretations (see Lemma 2.1 in [5]). Now the claim follows from Theorem 3 .

For Hamiltonicity we follow the same path. A well-known result of Dahlhaus [12] gives a first-order definable reduction from 3-SAT to Hamiltonicity that does not require any linear order on the input. However, that reduction is quadratic and would only achieve a lower bound of the form $k=\Omega(\sqrt{n})$ for $n$-vertex graphs. We work out a linear reduction:

Lemma 14. There exists $d>0$ such that, for every $k$ and every sufficiently large $n$, every $\mathrm{C}^{k}$-formula that defines the class of Hamiltonian graphs with $n$ vertices has $k \geq d n$.

Proof. In this case we need a minor modification of the textbook reduction from 3-SAT to the problem of deciding whether a graph is Hamiltonian in, e.g., 34]. As in the 3-colouring case, the output graph of the textbook reduction has one constant-size gadget for each constraint, and also one constant-size gadget for each variable. There is also one constant-size gadget for each variable-occurrence in the input 3-CNF formula, and three additional special vertices. This defines all the vertices of the graph. See Figure 9.7 of [34]. Since each clause contributes three variable occurrences, the total number of vertices is $O(n)+O(m)+O(m)$, where $n$ is the number of variables, and $m$ is the number of clauses of the input formula. However, for defining the edges of this reduction, one needs a linear order on the variables of the input formula for creating the path of variable gadgets in Figure 9.7 of [34]. Such a linear order is not available in our encoding (and cannot be available as otherwise the proof of Lemma 13 breaks down). Here is where we modify the construction. Instead of aligning the variable gadgets in the form of a path, we arrange them in the form of a big clique as in Figure 1 .

This modification does not introduce any new vertices and does not depend on any given ordering of the variables in the input formula. The analysis that proves that the reduction is correct is the same as in [34]. As in the proof of Lemma 13, it is not hard to see that this transformation is definable by a uniform quantifier-free interpretation, still without parameters or linear orders, and the result follows again from Theorem 3 and the closure of the logic under quantifier-free interpretations.

Proof of Theorem [2. Apply Theorem 3 and Lemmas 13 and 14 to Lemma 5, for LP lifts, and then to Lemma 1, for circuits, and in both cases use equation (26).

Lower Bound on the TSP Polytope As stated in the introduction, Yannakakis proved that the travelling salesman polytope does not have subexponential symmetric LP lifts. Here 


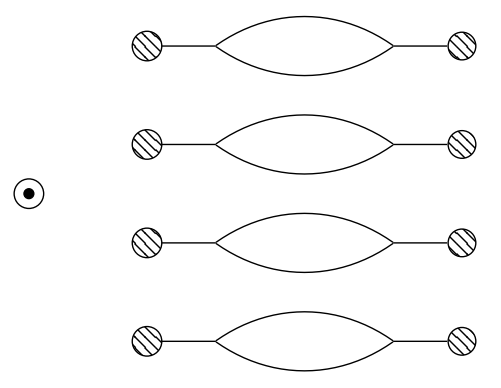

Figure 1: The variable gadgets arranged in the form of a big (unordered) clique. All striped vertices are connected by a big clique. The rightmost and leftmost non-striped vertices denote the entry and exit points, respectively, and they are also connected to all the striped vertices. These gadgets are connected with the rest of the graph as in Figure 9.7 of [34].

we show that this same lower bound follows from Theorem 2. In the next section we will see that the same type of argument cannot work for the matching polytope.

If $G=(V, E)$ is a graph with $V=[n]$, the incidence vector of $G$ is $\mathbf{x}^{G}=\left(x_{i j}^{G}: i, j \in[n]\right)$, the vector in $\mathbb{R}^{[n]^{2}}$ defined by $x_{i j}^{G}=1$ if $(i, j)$ is an edge of $G$, and $x_{i j}^{G}=0$ if $(i, j)$ is not an edge of $G$. Let $\mathrm{TSP}_{n}$ denote the convex hull of all the vectors of the form $\mathrm{x}^{C}$, where $C$ is a Hamilton cycle of the complete graph $\mathrm{K}_{n}$ on $n$ vertices. We want to show that every graphsymmetric LP lift that has $\operatorname{TSP}_{n}$ as shadow must be of exponential size as a consequence of Theorem 2.

Theorem 4 (Theorem 2 in [40]). Every graph-symmetric LP lift that has $\mathrm{TSP}_{n}$ as shadow has size $2^{\Omega(n)}$.

Proof. By Theorem 2, it suffices to show that if $\mathrm{TSP}_{n}$ were the shadow of a subexponentialsize graph-symmetric LP lift, then there would be a subexponential-size graph-symmetric LP lift that separates the $\mathbf{x}^{G}$ for which $G$ is Hamiltonian from the $\mathbf{x}^{G}$ for which $G$ is not Hamiltonian. Assume then that $P$ were symmetric LP lift of size $2^{o(n)}$ whose shadow is $\mathrm{TSP}_{n}$; let us say that its principal variables are $\mathbf{y}=\left(y_{i j}: i, j \in[n]\right)$ and that its auxiliary variables are $\mathbf{z}=\left(z_{k}: 1 \leq k \leq p(n)\right)$. Consider the following LP on the $x_{i j}, y_{i j}$ and $z_{k}$ variables:

$$
\begin{aligned}
& 0 \leq y_{i j} \leq x_{i j} \quad \text { for each } i, j \in[n] \\
& (\mathbf{y}, \mathbf{z}) \in P .
\end{aligned}
$$

Since by assumption $P$ is graph-symmetric, $Q$ is also graph-symmetric. Hence, it suffices to show that the projection of $Q$ on the $x$-variables separates Hamiltonian graphs from non-Hamiltonian graphs.

It is clear that if $G$ is a graph that contains a Hamilton cycle $C$, then $\mathbf{x}^{G}$ is in the projection of $Q$ on the $x$-variables: choose $\mathbf{y}=\mathbf{x}^{C}$, and let $\mathbf{z}$ witness its membership in $P$. Conversely, assume that $G$ is a graph and that $\mathbf{x}^{G}$ is in the projection of $Q$ on the $x$ variables. Then there exists $\mathbf{y}^{*}=\left(y_{i j}^{*}\right)_{i j}$ that is in the TSP polytope and satisfies the inequalities $0 \leq y_{i j}^{*} \leq x_{i j}^{G}$ for each $i, j \in[n]$. This means that the support of $\mathbf{y}^{*}$ defines a 
subgraph of $G$, and at the same time that $\mathbf{y}^{*}$ is a convex combination of Hamilton cycles of $\mathrm{K}_{n}$. Let $\mathbf{y}^{C}$ be one of the vectors in this convex combination, where $C$ is a Hamilton cycle of $\mathrm{K}_{n}$. The support of $\mathbf{y}^{C}$ is of course included in the support of $\mathbf{y}^{*}$, which means that $C$ is also a subgraph of $G$. So $G$ contains a Hamilton cycle and is thus Hamiltonian.

Upper bounds Let us start with the simple observation that the lower bounds of type $2^{\Omega(n)}$ on 3-colouring, 3-SAT and 3-XOR are optimal: all three cases can be solved by symmetric Boolean threshold circuits of size $2^{O(n)}$, and hence by symmetric LP lifts of size $2^{O(n)}$ by Lemma 1. Here $n$ is the number of vertices or variables, respectively. This follows from the fact that all three problems are definable in the existential fragment of monadic secondorder logic (i.e., monadic NP), which on structures of size $n$, straightforwardly translate into symmetric Boolean circuits of size $2^{O(n)}$. For Hamiltonicity, the straightforward symmetric upper bound is only $2^{O(n \log n)}$ on $n$-vertex graphs. It looks plausible that Bellman [9] and Held-Karp [22] dynamic programming $O\left(n^{2} 2^{n}\right)$ algorithm [9, 22] could be implemented in a symmetric Boolean circuit, but we are not aware of a reference where this has been worked out. What is known is that Hamiltonicity is not definable in (full) monadic second-order logic (see [17]).

The list of problems for which we proved a lower bound in Theorem 2 includes one that is decidable in polynomial time, i.e., 3-XOR. This means that any polynomial-size family of LP lifts or threshold circuits that recognizes 3-XOR must a fortiori be asymmetric. On the other hand, Theorem 1 says that any problem that is definable in FPC has polynomialsize symmetric LP-lifts. This includes graph planarity [20, any polynomial-time decidable property of graphs that exclude some minor [21], matrix singularity over rationals [10], solving systems of linear equations over rationals [25], and many others. By the results in [4], it also includes the problem of deciding whether a (general, not necessarily bipartite) graph contains a perfect matching. The family can even be taken to be polynomial-time uniform by applying the construction of Lemma 1 to the "easy half" of the equivalence between FPC and polynomial-size symmetric threshold circuits in [3].

Corollary 2. There is a (polynomial-time uniform) family of graph-symmetric LP lifts of polynomial size that recognizes the class of graphs that have a perfect matching.

This should be contrasted with the fact, proved by Yannakakis, that any symmetric LP lift of the perfect matching polytope $\mathrm{PM}_{n}$ has size $2^{\Omega(n)}$. Here, $\mathrm{PM}_{n}$ is defined as the convex hull of all vectors of the form $\mathbf{x}^{M}$, where $M$ is the edge set of a perfect matching of the complete graph $\mathrm{K}_{2 n}$ on $2 n$ vertices. Capturing $\mathrm{PM}_{n}$ by an LP lift or recognizing the class of graphs that have a perfect matching by an LP lift are different tasks. Both objects could be used for deciding whether a given graph has a perfect matching, but capturing $\mathrm{PM}_{n}$ has a demanding structural requirement that has no analogue in the other task. The upper bound of Corollary 2 also means that the argument that was used for deriving lower bounds for $\mathrm{TSP}_{n}$ in the proof of Theorem 4 cannot be adapted to $\mathrm{PM}_{n}$. Indeed, we do not know whether there is any route at all for deriving lower bounds for $\mathrm{PM}_{n}$ via our results.

In view of Corollary 2 one may wonder whether the convex hull of the incidence vectors of graphs that have a perfect matching has a small symmetric LP lift. This, however, is easily 
seen to not be the case: if it had, then its intersection with the halfplane $\sum_{i, j \in[2 n]: i \neq j} x_{i j}=2 n$ would be a small symmetric LP lift for $\mathrm{PM}_{n}$.

\subsection{Problems on Erdős-Rényi Random Graphs}

Let $\mathscr{G}(n, p)$ denote the Erdős-Rényi distribution on $n$-vertex labelled graphs with edge probability $p$. We write $G \sim \mathscr{G}(n, p)$ to mean that $G$ is a random graph distributed as in $\mathscr{G}(n, p)$. In this section we argue that, for average-case problems with respect to the uniform distribution $\mathscr{G}(n, 1 / 2)$, as well as for the type of problems that ask to distinguish $\mathscr{G}(n, 1 / 2)$ from some other distribution, polynomial-size symmetric LPs are as powerful as arbitrary not necessarily symmetric Boolean circuits. For average-case problems, this is indeed a direct consequence of our main result and the following well-known fact in descriptive complexity theory:

Theorem 5 (Corollary 4.8 in [23]). For every polynomial-time decidable class of graphs $\mathscr{C}$ there is an FPC-definable class of graphs $\mathscr{C}^{\prime}$ for which the probability that a random graph $G \sim$ $\mathscr{G}(n, 1 / 2)$ falls in the symmetric difference $\mathscr{C} \Delta \mathscr{C}^{\prime}$ is o(1).

The point of Theorem 5 is that the FPC formula that defines $\mathscr{C}^{\prime}$ does not require any order on the input graph, hence our Theorem 1 applies. Theorem 5 is indeed a consequence of the Immerman-Vardi Theorem [27, 38] and the fact that a linear order is, asymptotically almost surely on $\mathscr{G}(n, 1 / 2)$, definable in FPC. We return to this later. For the rest of this section let us focus our discussion on the problem of distinguishing $\mathscr{G}(n, 1 / 2)$ from some other distribution of random graphs, to which a direct application of Theorem 5 does not look possible.

We focus the discussion on the planted clique problem since it is one of the best studied such problems, although it will be clear from the discussion that the phenomenon is more general. Let $\mathscr{G}(n, p, k)$ denote the distribution that results from drawing a random graph from $\mathscr{G}(n, p)$ and then planting a random $k$-clique in it, i.e., adding the edges of a $k$-clique on a uniformly chosen subset of $k$ vertices. Following [8], the planted clique problem, also known as the hidden clique problem, comes in three flavours. Informally stated, these are:

- search: given $G \sim \mathscr{G}(n, p, k)$, find the planted clique,

- refutation: given $G \sim \mathscr{G}(n, p)$, certify that the clique number is less than $k$,

- decision: given $G \sim \mathscr{G}(n, p)$ or $G \sim \mathscr{G}(n, p, k)$, determine which is the case.

Formally, the decision version can be stated as follows. We say that a class of graphs $\mathscr{C}$ solves the decision version of the planted clique problem with parameters $p=p(n)$ and $k=k(n)$ and advantage $\epsilon=\epsilon(n)>0$ if for every large enough $n$ the following hold:

1. if $G \sim \mathscr{G}(n, p)$, then $G$ is in $\mathscr{C}$ with probability at least $1 / 2+\epsilon$,

2. if $G \sim \mathscr{G}(n, p, k)$, then $G$ is in $\mathscr{C}$ with probability at most $1 / 2-\epsilon$. 
Solvable in polynomial time (or in FPC, or by a family of LP-lifts, etc.) means that it is solvable by a class of graphs $\mathscr{C}$ that can be recognized in polynomial time (or in FPC, or by a family of LP-lifts, etc.). It should be clear that if the decision version is hard, then the other two versions of the problem can only be harder.

The planted clique problem is an easy-to-state average case problem that has a long history. Its formulation is attributed to Jerrum [29] and Kučera [32], independently. In the range $k(n)=\omega(\sqrt{n \log n})$, a simple algorithm based on degree sequences solves the search problem in polynomial time [32. In the range $k(n)=\Omega(\sqrt{n})$, a spectral based algorithm is known to solve the problem in polynomial time [2], but spectral-free algorithms are also known that run even in linear time [18]. For $k(n)=o(\sqrt{n})$ the status of the problem is famously open, even for its decision variant. Some lower bounds are known in restricted models, such as the original lower bound for Markov chain methods in [29]. Lower bounds are also known for (symmetric) linear and semidefinite program formulations of the problem. We discuss these next.

We formulate the clique problem on a graph $G=(V, E)$ as a non-linear polynomial optimization problem: For each vertex $v \in V$ introduce one variable $y_{v}$ that stands for the indicator whether $v$ belongs to the clique. The clique number is the maximum of $\sum_{v \in V} y_{v}$ subject to the constraints that $y_{u} y_{v}=0$ for each non-edge $(u, v) \notin E$, and $y_{v}^{2}-y_{v}=0$ for each $u \in V$. For the refutation and the decision versions of the problem, it is more natural to turn the objective function into a constraint $\sum_{v \in V} y_{v} \geq k$. This gives a different quadratic program feasibility problem for each graph $G$. Thinking of $G$ as given by the $\{0,1\}$-vector $\left(x_{u, v}\right)_{u, v \in[n]}$ in the usual way, the programs can be made uniform, i.e., a single quadratic program feasibility problem serves all graphs with $V=[n]$ :

$$
\begin{array}{ll}
\sum_{v \in[n]} y_{v} \geq k & \\
y_{u} y_{v} \leq x_{u, v} & \text { for } u, v \in[n] \\
y_{v}^{2}-y_{v}=0 & \text { for } v \in[n]
\end{array}
$$

While this a hard-to-solve quadratic program, there are several tractable relaxations that one can study. Two systematic methods for generating such relaxations were introduced by Lovász and Schrijver in [31, and Sherali and Adams in [37]. Both cases start by (naively) linearizing the quadratic program, i.e., replacing each quadratic term $y_{u} y_{v}$ in the constraints by a new variable $y_{\{u, v\}}$. The result is a very weak symmetric LP relaxations with auxiliary variables, but this is only the first level of the LS and SA hierarchies. The successive levels of the hierarchies are obtained by adding linear constraints that can be proved valid for the convex hull of solutions of the quadratic program by iterated applications of a simple rule of inference.

It can be seen that the levels of the hierarchies are always graph-symmetric LPs, with the $d$-th level having $n^{O(d)}$ auxiliary variables and constraints, where $n=|V|$. The strengths of the relaxations converge to the quadratic program in the sense that the polytopes they project to are tighter and tighter approximations of the convex hull of solutions of the quadratic program. Moreover, the exact convex hull is eventually reached no later than the $n$-th level. 
In order to appreciate the symmetry of the LPs that define the sucessive levels of the hierarchy, it useful to enter the details for the definition of the SA hierarchy. The first step in producing the $d$-th level of the SA hierarchy is to formally multiply each constraint of the quadratic program by an extended monomial of the form $\prod_{w \in I} y_{w} \prod_{w \in J}\left(1-y_{w}\right)$ for $I, J \subseteq V$ with $I \cap J=\emptyset$ and $|I \cup J| \leq d-1$. The second step is to expand out the formal expressions into sums of degree $d+1$ monomials, and multilinearize every monomial on the $y$-variables; note that this is a valid step only under the constraint $y_{v}^{2}-y_{v}=0$, hence for $\{0,1\}$-assignments. In the third step we introduce a new variable $y_{I}$ for each $I \subseteq V$ with $|I| \leq d+1$, and a new variable $y_{u, v, I}$ for each $u, v \in V$ and each $I \subseteq V$ with $|I| \leq d-1$. The fourth and final step is to linearize: replace each monomial $\prod_{w \in I} y_{w}$ by the new variable $y_{I}$, and each monomial $x_{u, v} \prod_{w \in I} y_{w}$ by the new variable $y_{u, v, I}$. The link between the two types of variables is established by setting $x_{u, v}=y_{u, v, \emptyset}$ and $y_{\emptyset}=1$. The symmetry of the resulting LP is obvious since the image $I^{(\pi)}$ of a set $I \subseteq V$ by a permutation $\pi \in \operatorname{Sym}_{V}$ has the same cardinality as $I$. The result is a graph-symmetric LP with $n^{O(d)}$ auxiliary variables and constraints, whose shadow eventually captures the convex hull of solutions of the quadratic program. Any level that approximates this hull well enough, even on average on $\mathscr{G}(n, 1 / 2)$, can be used for solving the planted clique problem.

The strengths and limitations of the LS and SA hierarchies (and beyond) for the planted clique problem have been the object of intensive study. Starting with the work of Feige and Krauthgamer [19], it is known that for each constant $d$, the $d$-th level of the LS hierarchy cannot distinguish between $\mathscr{G}(n, 1 / 2)$ and $\mathscr{G}(n, 1 / 2, k(n))$ when $k(n)=o(\sqrt{n})$ with any significant advantage. Their lower bound is much stronger than we stated it since it applies even to $\mathrm{LS}^{+}$, the semi-definite programming variant of the LS-hierarchy. For the SA hierarchy, the same result is attributed to the folklore, and more recent works [7, 26] have obtained analogous results for the even stronger Lasserre/Sums-of-Squares (SOS) hierarchies, also proving that the problem stays hard for their constant $d$ level when $k(n)=o(\sqrt{n})$. Further, in certain contexts, it is possible to prove that the Sherali-Adams hierarchy is optimal among symmetric LP lifts of comparable size. This includes a model of LP lifts for solving Boolean constraint satisfaction problems (see Theorem 4.1 in [11]). This means that, in such restricted contexts, proving lower bounds on the levels of Sherali-Adams is enough for getting size lower bounds for any symmetric LP lift.

In view of such success in proving lower bounds on the size of symmetric LP lifts, starting with Yannakakis, and including the discussion above on hierarchies for the planted clique problem, and also given our own lower bounds from Section 5.2, the following consequence of Theorem 1 may come as a surprise:

Corollary 3. If the planted clique problem with parameters $p=1 / 2$ and $k=k(n)$ is solvable in polynomial time with advantage $\epsilon>0$, then it is also solvable by a (polynomial-time uniform) family of polynomial-size graph-symmetric LP lifts with advantage $\epsilon-o(1)$.

In the rest of this section we show how to derive the descriptive complexity version of Corollary 3, from which Corollary 3 follows at once from Theorem 1. The descriptive complexity variant relies on the following well-known fact, which builds on the almost sure graph canonization methods of [6]: 
Theorem 6 (Theorem 4.6 in [23]). There is an FOC-formula $\phi(x, y)$ such that, for all $\epsilon>0$ and all sufficiently large $n$, if $G \sim \mathscr{G}(n, 1 / 2)$, then $\phi(x, y)$ defines a strict linear order on the vertices of $G$ with probability at least $1-\epsilon$.

We note that Theorem 6 is one of the two ingredients in the proof of Theorem 5 ; the second one is the Immerman-Vardi Theorem. While our proof uses only these two ingredients too, we do not see a direct way of getting it from Theorem 5 .

Theorem 7. If the planted clique problem with parameters $p=1 / 2$ and $k=k(n)$ is solvable in polynomial time with advantage $\epsilon>0$, then it is solvable in FPC with advantage $\epsilon-o(1)$.

Proof. Suppose that $\mathscr{C}$ is a polynomial time decidable class of graphs that solves the problem with advantage $\epsilon>0$. Let $\mathscr{C}^{\prime}$ denote the class of all ordered expansions of graphs in $\mathscr{C}$, i.e., the structures in $\mathscr{C}^{\prime}$ are finite structures over the vocabulary $L=\{E, R\}$, where $E$ and $R$ are binary relations symbols, and $E$ is interpreted as the edge relation of some graph in $\mathscr{C}$, and $R$ is interpreted as a strict linear order on its set of vertices. By the Immerman-Vardi Theorem, there is an FP formula $\psi$ that defines $\mathscr{C}^{\prime}$ on the class of all ordered graphs. Let $\phi(x, y)$ be the FOC formula from Theorem [6, and let $\theta$ be the conjunction of the following sentences:

$$
\begin{aligned}
& \forall x(\neg \phi(x, x)) \\
& \forall x \forall y \forall z(\neg \phi(x, y) \vee \neg \phi(y, z) \vee \phi(x, z)) \\
& \forall x \forall y(x=y \vee \phi(x, y) \vee \phi(y, x)) \\
& \psi[R / \phi] .
\end{aligned}
$$

This sentence says that $\phi$ defines a strict linear order and $\psi$ holds when each occurrence of $R$ is replaced by $\phi$. This is an FPC formula over the vocabulary of unordered graphs that defines a class $\mathscr{D}$ of graphs. We claim that $\mathscr{D}$ solves the problem with advantage $\epsilon-o(1)$.

By assumption and the fact that $\mathscr{C}^{\prime}$ contains all ordered expansions of graphs in $\mathscr{C}$ we have that the probability that some and hence every ordered expansion of $G$ satisfies $\psi$ is at least $1 / 2+\epsilon$ when $G \sim \mathscr{G}(n, 1 / 2)$, and at most $1 / 2-\epsilon$ when $G \sim \mathscr{G}(n, 1 / 2, k)$. Now, if $G \sim \mathscr{G}(n, 1 / 2)$, then the probability that $\phi$ does not define a linear order is $o(1)$, and the probability that some and hence every ordered expansion of $G$ satisfies $\psi$ is at least $1 / 2+\epsilon$, so the probability that $G$ satisfies $\theta$ is at least $1 / 2+\epsilon-o(1)$. On the other hand, if $G \sim \mathscr{G}(n, 1 / 2, k)$, then the probability that some and hence every ordered expansion of $G$ satisfies $\psi$ is at most $1 / 2-\epsilon$, so the probability that $G$ satisfies $\theta$ is even smaller, and $1 / 2-\epsilon \leq 1 / 2-\epsilon+o(1)$. It follows that $\mathscr{D}$ solves the problem with advantange $\epsilon-o(1)$.

\section{Concluding Remarks}

Our main result Theorem 1 establishes a tight three-way correspondence between symmetric Boolean threshold circuits, symmetric LP lifts, and bounded-variable formulas of counting logic. We used this to derive upper and lower bounds on the size of symmetric LPs and 
symmetric Boolean threshold circuits. We also used it to bound the asymmetric circuit complexity of the planted-clique problem by its symmetric LP lift complexity, up to a polynomial factor. There are several directions for further investigation that are suggested by this work.

The first one concerns the problem of circuits vs formulas. Composing the first inequality in the first item of Theorem 1 with the second inequality in the second item of Theorem 1, we get the result that, for every constant $k$, every symmetric Boolean threshold circuit of size at most $n^{k}$ translates into an equivalent $\mathrm{C}^{O(k)}$-formula of size $n^{O(k)}$. This is a size-efficient translation from circuits into formulas, albeit of different types: the source is a Boolean threshold circuit and the target is a counting logic formula. An explicit and uniform such translation is given in [3], and similarly, AND-OR-NOT circuits can translate into families of $\mathrm{L}^{k}$-formulas, where $\mathrm{L}^{k}$ stands for the $k$-variable fragment of first-order logic, without counting.

At first sight, the translation from circuits to formulas is unexpected as the circuit value problem is $\mathrm{P}$-complete, while the formula value problem is in $\mathrm{NC}^{1}$. However, as we noted, these are not Boolean formulas and the natural translation of formulas of $\mathrm{C}^{k}$ or $\mathrm{L}^{k}$ into circuits necessarily yields circuits with high fan-out. This also accords with the well-known fact that the combined complexity of the logic $\mathrm{L}^{k}$ is $\mathrm{P}$-complete for each $k \geq 3$ [39]. An intriguing question at this point is: What happens when we start the translation not from a circuit but from a symmetric Boolean (threshold) formula? Does this correspond to a natural syntactic fragment of the logic $\mathrm{L}^{k}\left(\right.$ or $\left.\mathrm{C}^{k}\right)$ ? Could such a translation shed light on the descriptive complexity of $\mathrm{NC}^{1}$ ? On the $\mathrm{NC}^{1}$ vs $\mathrm{P}$ question? Similar questions can be asked for symmetric bounded-depth threshold circuits, and other classes of circuits. It is worth noting that both Theorem [5] and Theorem 7 on the planted-clique problem scale all the way down to uniform $\mathrm{TC}^{0}$ and FOC. This is so because Theorem 6 gives a FOCformula and, in the presence of a linear order on the input, FOC captures uniform $\mathrm{TC}^{0}$ (see Proposition 12.6 in [28]). We view all this as motivation for finding a symmetric LP model of symmetric bounded-depth threshold circuits.

A different line of research that is suggested by our work relates to the computational complexity of the linear programming feasibility problem. It is well-known that this problem is P-complete under logspace reductions and a question posed in [4], where it was shown that this problem is in FPC, is whether it is complete for FPC under (say) FOC reductions. Our proof of Lemma 1 gives one route towards answering this question. The construction in the proof of the lemma should yield a first-order interpretation from the threshold circuit value problem to the LP feasibility problem. Since the known translations from FPC into symmetric threshold circuits are first-order interpretations themselves, the result should follow by composition. The FPC-completeness of the LP feasibility problem was also proved by Pakusa (unpublished manuscript), independently of our work. Pakusa's construction is very different from ours and raises the question whether the construction in his proof could yield a different proof of Lemma 1. His construction is inspired by the Sherali-Adams hierarchy of LP relaxations [37] (discussed in Section 51) and could well provide a more principled method than ours for constructing LP lifts with useful properties. 
Acknowledgments First author partially funded by European Research Council (ERC) under the European Union's Horizon 2020 research and innovation programme, grant agreement ERC-2014-CoG 648276 (AUTAR) and MICCIN grant TIN2016-76573-C2-1P (TASSAT3). The second author was partially supported by a Fellowship of the Alan Turing Institute under the EPSRC grant EP/N510129/1. The third author funded by the European Union's Horizon 2020 research and innovation programme under the Marie Skłodowska-Curie grant agreement No 795936. Some of the work reported here was initiated at the Simons Institute for the Theory of Computing during the programme on Logical Structures in Computation

in autumn 2016. We are grateful to Matthew Anderson for some very stimulating discussions on this topic. In particular, he suggested using Lemma 9 to prove the existence of supports. We also thank Wied Pakusa for sharing his manuscript on the FPC-completeness of linear programming with us.

\section{References}

[1] Noga Alon, Michael Krivelevich, and Benny Sudakov. Finding a Large Hidden Clique in a Random Graph. In Proceedings of the Ninth Annual ACM-SIAM Symposium on Discrete Algorithms, SODA '98, pages 594-598, Philadelphia, PA, USA, 1998. Society for Industrial and Applied Mathematics.

[2] Noga Alon, Michael Krivelevich, and Benny Sudakov. Finding a large hidden clique in a random graph. Random Structures \& Algorithms, 13(34):457-466, 1998.

[3] Matthew Anderson and Anuj Dawar. On Symmetric Circuits and Fixed-Point Logics. Theory of Computing Systems, 60(3):521-551, Apr 2017.

[4] Matthew Anderson, Anuj Dawar, and Bjarki Holm. Solving Linear Programs Without Breaking Abstractions. J. ACM, 62(6):48:1-48:26, December 2015.

[5] Albert Atserias and Anuj Dawar. Definable Inapproximability: New Challenges for Duplicator. In 27th EACSL Annual Conference on Computer Science Logic, CSL 2018, September 4-7, 2018, Birmingham, UK, pages 7:1-7:21, 2018.

[6] László Babai, Paul Erdös, and Stanley M. Selkow. Random Graph Isomorphism. SIAM J. Comput., 9(3):628-635, 1980.

[7] Boaz Barak, Samuel B. Hopkins, Jonathan A. Kelner, Pravesh Kothari, Ankur Moitra, and Aaron Potechin. A Nearly Tight Sum-of-Squares Lower Bound for the Planted Clique Problem. In FOCS, pages 428-437. IEEE Computer Society, 2016.

[8] Boaz Barak and David Steurer. Proofs, beliefs, and algorithms through the lens of sum-of-squares. Last accessed Jan 8, 2019, 2016.

[9] Richard Bellman. Dynamic Programming Treatment of the Travelling Salesman Problem. J. ACM, 9(1):61-63, January 1962. 
[10] Andreas Blass, Yuri Gurevich, and Saharon Shelah. Choiceless Polynomial Time. Ann. Pure Appl. Logic, 100(1-3):141-187, 1999.

[11] Siu On Chan, James R. Lee, Prasad Raghavendra, and David Steurer. Approximate Constraint Satisfaction Requires Large LP Relaxations. J. ACM, 63(4):34:1-34:22, 2016.

[12] Elias Dahlhaus. Reduction to NP-complete Problems by Interpretations. In Proceedings of the Symposium "Rekursive Kombinatorik" on Logic and Machines: Decision Problems and Complexity, pages 357-365, Berlin, Heidelberg, 1984. Springer-Verlag.

[13] Anuj Dawar. A Restricted Second Order Logic for Finite Structures. Inf. Comput., 143(2):154-174, 1998.

[14] Anuj Dawar. The nature and power of fixed-point logic with counting. ACM SIGLOG News, 2(1):8-21, 2015.

[15] Anuj Dawar and Pengming Wang. Definability of Semidefinite Programming and Lasserre Lower Bounds for CSPs. In LICS, pages 1-12. IEEE Computer Society, 2017.

[16] John D. Dixon and Brian Mortimer. Permutation Groups, volume 163 of Graduate Texts in Mathematics. Springer-Verlag New York, 1996.

[17] Heinz-Dieter Ebbinghaus and Jörg Flum. Finite model theory. Perspectives in Mathematical Logic. Springer, 1995.

[18] Uriel Feige and Robert Krauthgamer. Finding and certifying a large hidden clique in a semirandom graph. Random Structures \& Algorithms, 16(2):195-208, 2000.

[19] Uriel Feige and Robert Krauthgamer. The Probable Value of the Lovász-Schrijver Relaxations for Maximum Independent Set. SIAM J. Comput., 32(2):345-370, 2003.

[20] Martin Grohe. Fixed-Point Logics on Planar Graphs. In Thirteenth Annual IEEE Symposium on Logic in Computer Science, Indianapolis, Indiana, USA, June 21-24, 1998, pages 6-15, 1998.

[21] Martin Grohe. From polynomial time queries to graph structure theory. Commun. ACM, 54(6):104-112, 2011.

[22] Michael Held and Richard M. Karp. A Dynamic Programming Approach to Sequencing Problems. In Proceedings of the 1961 16th ACM National Meeting, ACM '61, pages 71.201-71.204, New York, NY, USA, 1961. ACM.

[23] Lauri Hella, Phokion G. Kolaitis, and Kerkko Luosto. Almost everywhere equivalence of logics in finite model theory. Bulletin of Symbolic Logic, 2(4):422-443, 1996.

[24] Wilfrid Hodges. Model Theory. Encyclopedia of Mathematics and its Applications. Cambridge University Press, 1993. 
[25] Bjarki Holm. Descriptive complexity of linear algebra. PhD thesis, University of Cambridge, UK, 2011.

[26] Samuel B. Hopkins, Pravesh Kothari, Aaron Henry Potechin, Prasad Raghavendra, and Tselil Schramm. On the Integrality Gap of Degree-4 Sum of Squares for Planted Clique. ACM Trans. Algorithms, 14(3):28:1-28:31, 2018.

[27] Neil Immerman. Languages that Capture Complexity Classes. SIAM J. Comput., 16(4):760-778, 1987.

[28] Neil Immerman. Descriptive complexity. Graduate texts in computer science. Springer, 1999.

[29] Mark Jerrum. Large Cliques Elude the Metropolis Process. Random Struct. Algorithms, $3(4): 347-360,1992$.

[30] Martin W. Liebeck. On Graphs Whose Full Automorphism Group is an Alternative Group or a Finite Classical Group. Proceedings of the London Mathematical Society, s3-47(2):337-362, 1983.

[31] László Lovász and Alexander Schrijver. Cones of Matrices and Set-Functions and 0-1 Optimization. SIAM Journal on Optimization, 1(2):166-190, 1991.

[32] Luděk Kučera. Expected complexity of graph partitioning problems. Discrete Applied Mathematics, 57(2):193 - 212, 1995. Combinatorial optimization 1992.

[33] Martin Otto. Bounded Variable Logics and Counting: A Study in Finite Models, volume 9 of Lecture Notes in Logic. Cambridge University Press, 2017.

[34] Christos H. Papadimitriou. Computational complexity. Academic Internet Publ., 2007.

[35] Thomas Rothvoss. The Matching Polytope has Exponential Extension Complexity. $J$. ACM, 64(6):41:1-41:19, 2017.

[36] Alexander Schrijver. Theory of linear and integer programming. Wiley-Interscience series in discrete mathematics and optimization. Wiley, 1999.

[37] Hanif D. Sherali and Warren P. Adams. A Hierarchy of Relaxations Between the Continuous and Convex Hull Representations for Zero-One Programming Problems. SIAM J. Discrete Math., 3(3):411-430, 1990.

[38] Moshe Y. Vardi. The Complexity of Relational Query Languages (Extended Abstract). In STOC, pages 137-146. ACM, 1982.

[39] Moshe Y. Vardi. On the Complexity of Bounded-Variable Queries. In PODS, pages 266-276. ACM Press, 1995.

[40] Mihalis Yannakakis. Expressing Combinatorial Optimization Problems by Linear Programs. J. Comput. Syst. Sci., 43(3):441-466, 1991. 